Pacific Journal of Mathematics

ON THE PRIME DIVISORS OF ZERO IN FORM RINGS 


\title{
ON THE PRIME DIVISORS OF ZERO IN FORM RINGS
}

\author{
L. J. RATLifF, JR.
}

\begin{abstract}
A number of new results concerning the prime divisors of zero in the form ring $\mathscr{F}(A, I)$ of a Noetherian ring $A$ with respect to an ideal $I$ in $A$ are found by using certain auxiliary rings of the Rees ring $\mathscr{R}(A, I)$. Then it is shown that in every semi-local ring $R$ there exist open ideals $Q$ such that the prime divisors of $u \mathscr{R}(R, Q)$ have a number of properties known to hold when $Q$ is a normal ideal and $R$ is analytically unramified.
\end{abstract}

1. Introduction. Form rings ( = associated graded rings) have been studied in a number of important papers (such as [3], where the concept was introduced, and $[7,15,16])$ and many textbooks on commutative algebra (such as $[\mathbf{1 , 5 , 2 1 ]})$. Such rings are an important tool in many investigations in commutative algebra, and therein they appear in an auxiliary role. Our concern in this paper is with a certain aspect of the form rings themselves, namely, their prime divisors of zero. It is important to know about these prime ideals for a number of reasons, such as: they can be used to help determine when a local ring is quasi-unmixed [10, Theorem 3.8(2)]; unmixed [5, (25.1)]; analytically irreducible [5, (25.15)]; or, analytically normal [5, (25.15)]. (Also, if $I$ and $K$ are ideals in a Noetherian ring $A$ and $\bar{K}$ is the $I$-form ideal of $K$ in $\mathscr{F}=\mathscr{F}(A, I)$ (the form ring of $A$ with respect to $I)$, then $\mathscr{F} / \bar{K} \cong$ $\mathscr{F}(A / K,(I+K) / K)$, so knowledge about prime divisors of zero in form rings immediately gives knowledge about the prime divisors of $\bar{K}$.)

It turns out that, due to an important result of D. Rees [15, Theorem $2.1]$, the study of the prime divisors of zero in $\mathscr{F}(A, I)$ is equivalent to studying the prime divisors of the principal ideal $u \mathscr{R}$ in the Rees ring $\mathscr{R}=\mathscr{R}(A, I)$, since $\mathscr{F} \cong \mathscr{R} / u \mathscr{R}$ (and in this isomorphism, $\bar{K} \cong$ $(K A[t, u] \cap \mathscr{R}, u) \mathscr{R})$. Now from this point of view, a number of auxiliary rings can be brought into play to help determine properties of the prime divisors of $u \mathscr{R}$ (and vice versa, these properties imply certain results for the auxiliary rings). (And because of this, the results in this paper are stated for the prime divisors of $u \mathscr{R}$, and the corresponding results for the prime divisors of zero in the form rings (or the prime divisors of $\bar{K}$ ) are not explicitly stated.)

The specific auxiliary rings which are of importance below are $\mathscr{R}\left(A, I^{n}\right)$ (for $\left.n \geqq 1\right), \mathscr{R}\left(A^{*}, I A^{*}\right)$ (where $A$ is semi-local and $A^{*}$ is its 
completion), and the integral closures of these rings. By using these auxiliary rings, it is seen that there are certain one-to-one correspondences and certain subspace properties between the prime divisors of $u \mathscr{R}$ and the prime divisors of $(u)$ in these auxiliary rings. To explain this in a little more detail, a brief summary of the results in this paper will now be given.

Section 2 contains a few preliminary definitions and results which are needed through $\$ 5$.

In $\S 3$, it is shown that $u \mathscr{R}\left(A, I^{n}\right)$ has at most as many minimal prime divisors as $u \mathscr{R}(3.2)$, where $\mathscr{R}=\mathscr{R}(A, I)$, and that there is a one-to-one correspondence between the prime divisors of $(u)$ in the integral closures of these rings (3.3). Also, two additional important results are given in (3.6) and (3.7). The first shows that if $I$ is not nilpotent, then, for each prime divisor $p^{\prime}$ of $u \mathscr{R}^{\prime}\left(\mathscr{R}^{\prime}=\right.$ the integral closure of $\left.\mathscr{R}\right), p^{\prime} \cap \mathscr{R}$ is a relevant prime divisor of $u \mathscr{R}$ (that is, not all homogeneous elements of positive degree are in $\left.p^{\prime} \cap \mathscr{R}\right)$. The other shows that if $I \subseteq J$, the Jacobson radical of $A$, then the depth of each homogeneous ideal $H$ in $\mathscr{R}$ can be computed thru some maximal homogeneous prime ideal in $\mathscr{R}$ (so this holds, in particular, for the prime divisors of $u \mathscr{R}$ ).

Section 4 is concerned with the prime divisors of $(u)$ in $\mathscr{R}=\mathscr{R}(R, I)$, where $I$ is an arbitrary ideal in a semi-local ring $R$, and it is shown that there is a many-one correspondence between the prime divisors $p^{0}$ of $(u)$ in $\mathscr{R}^{0}=\mathscr{R}\left(R^{*}, I R^{*}\right)$ and the prime divisors $p$ of $(u)$ in $\mathscr{R}$ and, if $p^{0}$ and $p$ correspond, then $\mathscr{R}_{p}$ is a subspace of $\mathscr{R}_{p^{0}}^{0}(4.2)$. Also, certain relationships between the depths of the prime divisors of $(u)$ in $\mathscr{R}$ and $\mathscr{R}^{0}$ and the depths of the prime divisors of $(0)$ in $R^{*}$ are given in (4.3). Then, similar results are shown to hold for the prime divisors of $(u)$ in the integral closures of $\mathscr{R}$ and $\mathscr{R}^{0}$ (see (4.5) and (4.6)).

If $I$ is an open ideal in $R$, then it is shown in $\S 5$ that the results of $\S 4$ can be considerably sharpened. For example, the correspondence between the prime divisors of $(u)$ in $\mathscr{R}$ and $\mathscr{R}^{0}$ is one-to-one, and then depth $p=$ depth $p^{0}$ and $\mathscr{R}_{p}$ is a dense subspace of $\mathscr{R}_{p^{0}}^{0}$ (5.1.1). Again, related results hold for the integral closures of $\mathscr{R}$ and $\mathscr{R}^{0}$ (see (5.1.3) and (5.1.4)).

Section 6 contains some additional preliminary results (on normal ideals and $q$ - $v$-rings) which are needed to prove the results in $\$ 7$.

In (7.1), it is shown that in every semi-local ring $R$ there exist open ideals $B$ such that the prime divisors of $(u)$ in $\mathscr{S}=\mathscr{R}(R, B)$ have many properties in common with the case when $R$ is analytically unramified and $B$ is a normal ideal (see the paragraph preceding (7.1)). Then it is shown that there exists a one-to-one correspondence between the minimal prime divisors of $(u)$ in $\mathscr{S}, \mathscr{S}^{0}, \mathscr{S}^{\prime}, \mathscr{S}^{0 \prime}$, and $\mathscr{R}\left(R, B^{k}\right)$, for all $k \geqq 1$ (7.4) and (7.6).

Section 8 contains a few applications of the results in this paper to: 
semi-local rings which are analytically unramified (8.1), analytically irreducible (8.3), and quasi-unmixed ((8.4) and (8.5)); and, the (catenary) chain conjectures (8.6). Then this paper is closed by asking three questions in $\$ 9$.

2. Preliminary definitions and known facts. In this paper, all rings are assumed to be commutative with an identity, and the terminology is, in general, the same as that in [5]. However, a number of the concepts that are needed are, perhaps, not too well known. For this reason, in this section we define some of these concepts and list the facts concerning them that are needed through $\$ 5$. (Section 6 contains additional definitions and facts that are needed in §7.) We begin with the definition of Rees rings.

(2.1) Definition. Let $I=\left(b_{1}, \cdots, b_{g}\right) A$ be an ideal in a Noetherian ring $A$, let $t$ be an indeterminate, and let $u=1 / t$. Then the Rees ring $\mathscr{R}=\mathscr{R}(A, I)$ of $A$ with respect to $I$ is the subring $\mathscr{R}=A\left[t b_{1}, \cdots, t b_{g}, u\right]$ of $A[t, u]$.

It is clear from (2.1) that the elements in $\mathscr{R}$ are finite sums $\sum_{-m}^{n} c_{i} t^{i}$, where $c_{i} \in I^{i}$ (with the convention that $I^{i}=A$, for $i \leqq 0$ ), so $\mathscr{R}$ is a graded Noetherian subring of $A[t, u]$. (2.2) lists a number of other known facts concerning Rees rings of a Noetherian ring which will be needed in what follows. (Most of the results are not proved in this generality in the references, but the proof of the more general case is the same.)

(2.2) Remark. Let $I=\left(b_{1}, \cdots, b_{g}\right) A$ be an ideal contained in the Jacobson radical of a Noetherian ring $A$, let $\mathscr{R}=\mathscr{R}(A, I)$, and let $B^{*}$ denote the ideal $B A[t, u] \cap \mathscr{R}$, where $B$ is an ideal in $A$. Then the following statements hold:

(2.2.1) [14, p. 229]. $u$ is a regular element in $\mathscr{R}$ and $u^{n} \mathscr{R} \cap A=I^{n}$, for all $n \geqq 1$. Moreover, the maximal homogeneous ideals in $\mathscr{R}$ are the ideals $\left(u, M^{*}\right) \mathscr{R}=\left(u, M, t b_{1}, \cdots, t b_{g}\right) \mathscr{R}$, where $M$ is a maximal ideal in $A$.

(2.2.2) [15, Theorem 1.5]. If $\cap q_{i}$ is an irredundant primary decomposition of an ideal $B$ in $A$, where $q_{\imath}$ is $p_{\imath}$-primary, then $p_{\imath}^{*}$ is prime, $q_{i}^{*}$ is $p_{i}^{*}$-primary, and $\cap q_{i}^{*}$ is an irredundant primary decomposition of $B^{*}$.

(2.2.3) $\left[15\right.$, Lemma 1.1]. $\mathscr{R}(A, I) / B^{*} \cong \mathscr{R}(A / B,(I+B) / B)$. 
(2.2.4) $\left[10\right.$, Remark 3.7]. Height $B^{*}=$ height $B$ and altitude $\mathscr{R}=$ altitude $A+1=$ height $\left(u, M^{*}\right) \mathscr{R}$, for each maximal ideal $M$ in $A$ such that height $M=$ altitude $A$. Therefore, by (2.2.3), depth $B^{*}=$ height $\left(u, M^{*}\right) \mathscr{R} / B^{*}=\operatorname{depth} B+1$, where $M$ is a maximal ideal in $A$ such that depth $B=$ height $M / B$.

(2.2.5) [15, Theorem 2.1]. $\mathscr{R} / u \mathscr{R} \cong \mathscr{F}(A, I)$, the form ring of $A$ with respect to $I$.

Although (2.2.5) is proved only for an $M$-primary ideal in a local ring $(R, M)$ in [15], exactly the same proof shows that $(2.2 .5)$ holds, in fact, for an arbitrary ideal $I$ in an arbitrary Noetherian ring $A$. It is because of this that Rees rings will be of considerable importance in this paper. For, this shows that all the information concerning the number of prime divisors of zero, their depths, etc. in the form rings $\mathscr{F}(A, I)$ of $A$ with respect to $I$ can be obtained from the corresponding Rees rings $\mathscr{R}(A, I)$. Also, Rees rings are easier to work with, and even more information can be obtained by working with the Rees rings rather than the form rings (such as certain one-to-one correspondences and certain subspace properties; for example see (4.2.1) and (5.1.1)). Therefore, most of the results in this paper will be concerned with the rings $\mathscr{R}(A, I)$ - and the corresponding results for the form rings will usually not be explicitly stated.

It will frequently be necessary to pass from a ring to its integral closure or its completion. Therefore we adopt the following two notational conventions, in order to avoid continual repetitions.

(2.3) Notation. (2.3.1) $A^{\prime}$ denotes the integral closure of a ring $A$ in its total quotient ring.

(2.3.2) $R^{*}$ denotes the $J$-adic completion of a semi-local ring $R$, where $J$ is the Jacobson radical of $R$.

Since $u \mathscr{R}$ and $u \mathscr{R}^{\prime}$ are principal ideals generated by a regular element, such ideals will be of importance in what follows. Many facts concerning the prime divisors of such an ideal are well known. Two of the less well known such facts which will be needed through $\$ 5$ are given in the following remark.

(2.4) Remark. Let $b$ be a regular element in $A^{\prime}$, where $A$ is a Noetherian ring. Then the following statements hold:

(2.4.1) [11, Proposition 2.13]. $\quad b A^{\prime}$ is a finite intersection of height one primary ideals, and if $p$ is a prime divisor of $b A^{\prime}$, then $p$ contains exactly one minimal prime ideal $z$ and $A_{p} / z A_{p}$ is a DVR (discrete valuation ring). 
(2.4.2) [11, Theorem 2.15]. If $C$ is a (not necessarily Noetherian) ring such that $A \subseteq C \subseteq A^{\prime}$ and $p$ is a prime divisor of $b A^{\prime}$, then $p \cap C$ is a prime divisor of each principal ideal generated by a regular element which it contains.

One further definition and two facts concerning it are still needed.

(2.5) Definition. Let $I$ be an ideal in a ring $A$. Then an element $x \in A$ is integrally dependent on $I$ in case $x$ satisfies an equation of the form $x^{n}+c_{1} x^{n-1}+\cdots+c_{n}=0$, where $c_{J} \in I^{\prime}(j=1, \cdots, n)$ [5, Exercises, p. 34]. The set $I_{a}$ of elements in $A$ which are integrally dependent on $I$ is the integral closure of $I$ in $A . \quad I$ is integrally closed in case $I_{a}=I$.

(2.6) REMARK. The following statements hold for an ideal $I$ in a ring $A$ :

(2.6.1) $[8, \S 6] . \quad I \subseteq I_{a} \subseteq \operatorname{Rad} I$ and $I_{a}$ is an ideal in $A$ such that $\left(I_{a}\right)_{a}=I_{a}$.

(2.6.2) If $A$ is Noetherian, then

$$
\mathscr{R}(A, I)^{\prime}=A^{\prime}\left[u, t B_{a}, \cdots, t^{\prime}\left(B^{\imath}\right)_{a}, \cdots\right],
$$

where $B=I A^{\prime}$.

Proof. (2.6.2) If $c \in\left(B^{k}\right)_{a}$, then by multiplying an equation of integral dependence of $c$ on $B^{k}$ (say of degree $n$ ) by $t^{n k}$, it is readily seen that $c t^{k} \in \mathscr{R}\left(A^{\prime}, I A^{\prime}\right)^{\prime}$, so it follows that $A^{\prime}\left[u, t B_{a}, \cdots\right] \subseteq \mathscr{R}\left(A^{\prime}, I A^{\prime}\right)^{\prime}=$ $\mathscr{R}(A, I)^{\prime}$. On the other hand, if $r \in A^{\prime}$ and $r t^{n} \in \mathscr{R}(A, I)^{\prime}$, then, from an equation of integral dependence, it is seen that $r \in\left(I^{n} A^{\prime}\right)_{a}$, so $r t^{n} \in A^{\prime}\left[u, t B_{a}, \cdots\right] . \quad$ (2.6.2) follows from this, since $\mathscr{R}(A, I)^{\prime}$ is a graded subring of $A^{\prime}[t, u]$.

3. Prime divisors of zero in $\mathscr{F}(A, I)$. The results in this section are concerned with the prime divisors of zero in the form ring $\mathscr{F}(A, I)$, where $I$ is an arbitrary ideal in an arbitrary Noetherian ring $A$. Actually, as noted after (2.2), the results are specifically concerned with the prime divisors of $u \mathscr{R}(A, I)$. It is shown that knowledge of the prime divisors of $u \mathscr{R}\left(A, I^{n}\right)$ (with $n \geqq 2$ ) and of $u\left(\mathscr{R}(A, I)^{\prime}\right)$ give some information concerning the prime divisors of $u \mathscr{R}(A, I)$, and vice versa. We begin with the following result.

(3.1) Proposition. Let I be an ideal in a Noetherian ring $A$, fix a positive integer $n$, and let $\mathscr{R}=\mathscr{R}(A, I)$ and $\mathscr{P}=\mathscr{R}\left(A, I^{n}\right)$. Then $S_{1}=$ 
$\{$ depth $q ; q$ is a minimal prime divisor of $u \mathscr{P}\} \subseteq S_{2}=\{$ depth $p$; $p$ is a minimal prime divisor of $u \mathscr{R}\} \subseteq S_{3}=\{$ depth $Q ; Q$ is a prime divisor of $u \mathscr{P}\}$.

Proof. Let $q$ be a minimal prime divisor of $u \mathscr{P}$, and let $\mathscr{A}=$ $A\left[t^{n} I^{n}, u^{n}\right]$. Then $\mathscr{A} \cong \mathscr{P}$, so there exists a minimal prime divisor $q^{\prime}$ of $u^{n} \mathscr{A}$ that corresponds to $q$ under the isomorphism, and then depth $q^{\prime}=$ depth $q$. Also, $\mathscr{A} \subseteq \mathscr{R}$ and $\mathscr{R}$ is integral over $\mathscr{A}$, so there exists a minimal prime divisor $p$ of $u \mathscr{R}$ such that $p \cap \mathscr{A}=q^{\prime}$, and necessarily depth $p=$ depth $q^{\prime}$. Therefore $S_{1} \subseteq S_{2}$.

Let $p$ be a minimal prime divisor of $u \mathscr{R}$. Now $\mathscr{R}$ is integral over $\mathscr{A}[u]$ and $\mathscr{A}[u]$ and $\mathscr{R}$ have the same total quotient ring. Therefore, since height $p=1, p^{\prime}=p \cap \mathscr{A}[u]$ is a prime divisor of $u^{n} \mathscr{A}[u]$, by (2.4.2). Also, $\mathscr{A}[u]$ is a free $\mathscr{A}$-module (since $u$ is transcendental over $A)$, so $p \cap \mathscr{A}=p^{\prime} \cap \mathscr{A}$ is a prime divisor of $u^{n} \mathscr{A}$ and depth $p=\operatorname{depth}$ $p \cap \mathscr{A}$. Therefore, by the isomorphism noted above, there exists a prime divisor $Q$ of $u \mathscr{P}$ such that depth $Q=\operatorname{depth} p$, hence $S_{2} \subseteq S_{3}$.

(3.2) REMARK. It was actually shown in the first paragraph of the proof of (3.1) that if $d$ is a given positive integer and $u \mathscr{P}$ has exactly $k$ minimal prime divisors of depth $=d$, then $u \mathscr{R}$ has at least $k$ minimal prime divisors of depth $=d$.

It follows from (3.2) that if $u \mathscr{R}$ has only one minimal prime divisor, then this also holds for $u \mathscr{P}$. Concerning this, see (5.3).

The next proposition shows that a considerably sharper result than (3.1) holds for the prime divisors of $(u)$ in $\mathscr{R}^{\prime}$ and $\mathscr{P}^{\prime}$. (Besides being of interest in its own right, (3.3) will be of importance in a number of proofs below.)

(3.3) Proposition. With $A, I, \mathscr{R}$, and $\mathscr{P}$ as in (3.1), there exists a one-to-one correspondence between the (minimal) prime divisors $p^{\prime}$ of $u \mathscr{R}^{\prime}$ and the (minimal) prime divisors $q^{\prime}$ of $u \mathscr{P}^{\prime}$ such that if $p^{\prime}$ and $q^{\prime}$ correspond, then depth $p^{\prime}=$ depth $q^{\prime}$.

Proof. (Note that the prime divisors of $u \mathscr{R}^{\prime}$ and of $u \mathscr{P}^{\prime}$ are all height one, by (2.4.1).) Let $\mathscr{A}=A\left[t^{n} I^{n}, u^{n}\right]$, so $\mathscr{A} \cong \mathscr{P}$, hence $\mathscr{A}^{\prime} \cong \mathscr{P}^{\prime}$. Therefore it suffices to show that there exists a one-to-one correspondence between the prime divisors of $u \mathscr{R}^{\prime}$ and the prime divisors of $u^{n} \mathscr{A}^{\prime}$ such that corresponding ideals have the same depth. For this, note that $\mathscr{A}^{\prime} \subseteq \mathscr{R}^{\prime}$ and $\mathscr{R}^{\prime}$ is integral over $\mathscr{A}^{\prime}$, so if $q$ is a prime divisor of $u^{n} \mathscr{A}^{\prime}$, then there exists a prime divisor $p^{\prime}$ of $u \mathscr{R}^{\prime}$ such that $p^{\prime} \cap \mathscr{A}^{\prime}=q$ and depth $p^{\prime}=$ depth $q$.

On the other hand, if $p^{\prime}$ is a prime divisor of $u \mathscr{R}^{\prime}$, then let $B=I A^{\prime}$ 
and $c \in\left(B^{k}\right)_{a}$ (for some $k \geqq 0$ ) such that $c t^{k} \in p^{\prime}$ and $c t^{k}$ isn't in any other prime divisor of $u \mathscr{R}^{\prime}$. (Note that $\mathscr{R}^{\prime}$ is a graded subring of $A^{\prime}[t, u]$ (so the prime divisors of $u \mathscr{R}^{\prime}$ are homogeneous), and $\mathscr{R}^{\prime}=$ $A^{\prime}\left[u, t B_{a}, \cdots, t^{\prime}\left(B^{i}\right)_{a}, \cdots\right]$ and $\mathscr{A}^{\prime}=A^{\prime}\left[u^{n}, t^{n}\left(B^{n}\right)_{a}, \cdots, t^{n i}\left(B^{n i}\right)_{a}, \cdots\right]$, by (2.6.2).) Then $c^{n} t^{n k} \in p^{\prime} \cap \mathscr{A}^{\prime}$ and $c^{n} t^{n k} \notin p^{\prime \prime} \cap \mathscr{A}^{\prime}$, for all other prime divisors $p^{\prime \prime}$ of $u \mathscr{R}^{\prime}$. Also, $p^{\prime} \cap \mathscr{A}^{\prime}$ is a prime divisor of $u^{n} \mathscr{A}^{\prime}$ (as in the last paragraph of the proof of (3.1)) and depth $p^{\prime}=\operatorname{depth} p^{\prime} \cap \mathscr{A}^{\prime}$.

(3.4) Corollary. With the notation of (3.1), \{depth $p^{\prime} ; p^{\prime}$ is a (minimal) prime divisor of $\left.u \mathscr{R}^{\prime}\right\}=\left\{\right.$ depth $q^{\prime} ; q^{\prime}$ is a (minimal) prime divisor of $\left.u \mathscr{P}^{\prime}\right\} \subseteq\{$ depth $P ; P$ is a prime divisor of $u \mathscr{R}\} \cap\{$ depth $Q ; Q$ is a prime divisor of $u \mathscr{P}\}$.

Proof. The first two sets are equal by (3.3), and it is contained in each of the last two sets, since if $p^{\prime}$ is a prime divisor of $u \mathscr{R}^{\prime}$, then $p^{\prime} \cap \mathscr{R}$ is a prime divisor of $u \mathscr{R}(2.4 .2)$ and depth $p^{\prime} \cap \mathscr{R}=$ depth $p^{\prime}$ (and a similar statement holds for the prime divisors of $u \mathscr{P}^{\prime}$ ).

The next remark is concerned with $u \mathscr{R}\left(A, I_{a}\right)$. (Considerably more will be said about the prime divisors of this ideal when $A$ is semi-local and $I$ is an open ideal, in $\S 7$.)

(3.5) Remark. With $A, I$, and $\mathscr{R}$ as in (3.1), $k_{1} \leqq k_{2} \leqq k_{3}$, there $k_{1}$ (resp., $k_{2}, k_{3}$ ) is the number of minimal prime divisors of $u \mathscr{R}$ (resp., $\left.u \mathscr{R}\left(A, I_{a}\right), u \mathscr{R}^{\prime}\right)$.

Proof. This follows from integral dependence, since $\mathscr{R} \subseteq$ $\mathscr{R}\left(A, I_{a}\right) \subseteq \mathscr{R}^{\prime}$.

The next result is concerned with relevant prime divisors of $u \mathscr{R}$. To give one reason why these are of interest, the following terminology is needed.

A homogeneous ideal $H$ in a graded ring is said to be irrelevant if it contains all homogeneous elements of sufficiently large degree; otherwise, $H$ is said to be relevant. If all prime divisors of zero in a form ring $\mathscr{F}(A, I)$ are relevant, then $I$ has very strongly superficial elements of all large degree $k$; that is, there exist $x \in I^{k}$ such that $I^{n+k}: x A=I^{n}$, for all $n \geqq 0$. (See [20, Theorem 2.5].) (3.6) shows that at least the prime divisors $p^{\prime} \cap \mathscr{R}$ of $u \mathscr{R}$ are relevant, if height $I>0$.

I am indebted to the referee for the following proof of (3.6), which is simpler than my original proof. 
(3.6) Proposition. With $A, I$, and $\mathscr{R}$ as in (3.1), if height $I>0$, then, for each (minimal) prime divisor $p^{\prime}$ of $u \mathscr{R}^{\prime}, t I \not \subset p^{\prime} \cap \mathscr{R}$.

Proof. Let $p^{\prime}$ be a prime divisor of $u \mathscr{R}^{\prime}$, so there exists a unique minimal prime ideal $z^{\prime}$ in $\mathscr{R}^{\prime}$ such that $z^{\prime} \subset p^{\prime}$, and then $\left(\mathscr{R}_{p^{\prime}}^{\prime}\right) / z^{\prime} \mathscr{R}_{p^{\prime}}^{\prime}$ is a DVR (2.4.1). Therefore $\mathscr{R}_{p^{\prime}}^{\prime}$ defines a valuation $v$ on the total quotient ring of $\mathscr{R}^{\prime}\left(v(x)=\infty\right.$ if and only if $x=c / b$ with $b, c \in \mathscr{R}^{\prime}$ and $\left.c \in z^{\prime}\right)$.

Assume height $I>0$ (so $I \not \subset z^{\prime}$ ), and suppose that $t I \subseteq$ $p^{\prime} \cap \mathscr{R}$. Then $t I \subseteq p^{\prime}$, so $v(u)<v(I)=v\left(\left(I A^{\prime}\right)_{a}\right)$, where $v(I)=$ $\min \{v(x) ; x \in I\}<\infty$ (since $I \not \subset z^{\prime}$ ). Define a homogeneous valuation $v^{\prime}$ on $\mathscr{R}^{\prime}$ by $v^{\prime}\left(b t^{r}\right)=v(b)-r v(I)$, where $b \in\left(I^{r} A^{\prime}\right)_{a}$ (see (2.6.2)). Then $v^{\prime}\left(b t^{r}\right) \leqq v\left(b t^{r}\right)$, if $r \geqq 0$, and $v\left(b t^{r}\right)>0$, if $r<0$, so the center of $v^{\prime}$ on $\mathscr{R}^{\prime}$ is contained in $p^{\prime}$ and $v^{\prime}(u)>0$. It follows that $v^{\prime}$ has center $p^{\prime}$ on $\mathscr{R}^{\prime}$, and $v^{\prime}$ is extendable to $\mathscr{R}_{p^{\prime}}^{\prime}$, hence $v^{\prime}$ coincides with $v$. But this is a contradiction, since $v^{\prime}(x)=0$, for some $x \in t I$. Therefore $t I \not \subset p^{\prime}$.

The final proposition in this section shows the very important (and somewhat surprising) result that the depth of a homogeneous ideal in $\mathscr{R}$ can be computed thru a maximal homogeneous prime ideal in $\mathscr{R}$. An important corollary concerning the prime divisors of zero in $\mathscr{R}$ is given in (3.8).

(3.7) Proposition. With $A, I$, and $\mathscr{R}$ as in (3.1), assume that $I$ is contained in the Jacobson radical of $A$. Then, for each homogeneous ideal $H$ in $\mathscr{R}$ such that depth $H<\infty$, there exists a maximal homogeneous prime ideal $\mathcal{M}$ in $\mathscr{R}$ such that $H \subseteq \mathcal{M}$ and depth $H=$ height $M / H$.

Proof. Since each prime divisor of $H$ is homogeneous, it clearly suffices to prove this in the case that $H=P$ is a homogeneous prime ideal of finite depth. If $I$ is nilpotent, then $t I \subseteq P$, so either $\mathscr{R} / P=$ $A /(P \cap A)$ (if $u \in P)$ or $u+P$ is transcendental over $A /(P \cap A)$ (if $u \notin P$, since $P=(P \cap A) A[t, u] \cap \mathscr{R}$, as is shown below). The conclusion readily follows in either case from (2.2.1).

Therefore assume that $I$ isn't nilpotent. If $u \notin P$, then let $p=$ $P \cap A$ and $Q=p A[t, u] \cap \mathscr{R}$. Then $P=Q, \quad$ as will now be shown. Since $u \notin P, p A[t, u] \subseteq P A[t, u]$, so $Q \subseteq P$. Also, $P$ and $Q$ are homogeneous, so it suffices to show that $Q_{[i]}=P_{[i]}(-\infty<i<\infty)$, where, for a homogeneous ideal $C$ in $\mathscr{R}, C_{[i]}=\left\{a \in A ; a t^{i} \in C\right\}$. Since $Q \subseteq P, Q_{[i]} \subseteq P_{[i]}$, for all $i$. Also, $P_{[i]} \subseteq Q_{[i]}$, for $i \leqq 0$, since if $a \in P_{[i]}$ $(i \leqq 0)$, then $a u^{i} \in P$ and $u \notin P$, so $a \in P \cap A=Q \cap A \subseteq Q$, hence $a u^{i} \in Q$, and so $a \in Q_{[i]}$. Finally, it is readily seen that, if $i>0$, then $Q_{[i]}=p \cap I^{i}$ and $P_{[i]}=u^{\prime} P \cap A \subseteq(P \cap A) \cap I^{i}$, so $P_{[i]} \subseteq Q_{[i]}$. Therefore $P=Q$, so the conclusion follows from (2.2.4). 
Thus it may be assumed that $u \in P$. Let $N$ be a maximal ideal in $\mathscr{R}$ such that depth $P=$ height $N / P=$ (say) $d$. Let $P=P_{0} \subset P_{1} \subset \cdots \subset P_{d}=$ $N$ be a saturated chain of prime ideals in $\mathscr{R}$ between $P$ and $N$, and let $i$ be the largest subscript such that $P_{1}$ is homogeneous. Then it may clearly be assumed that $i<d$. Let $b \in I$ such that $t b \notin N$. (Since $u \in N$, if $t I \subseteq N$, then $\mathscr{R} / N=A /(N \cap A)$, so $M=N \cap A$ is maximal and $N=(u, t I, M) \mathscr{R}$ is homogeneous.) Assume temporarily that $b$ is regular in $R$. Let $\mathscr{S}=\mathscr{R}[1 / t b]$ and $B=A[I / b]$, so $\mathscr{S}=B[t b, 1 / t b]$ and $t b$ is transcendental over $B$. Let $p_{\jmath}=P_{,} \mathscr{S} \cap B(j=0,1, \cdots, d)$. Then, by [10, Remark 3.11]: $Q_{l}=p_{1} \mathscr{S} \cap \mathscr{R}$ is homogeneous; $Q_{1} \subseteq P_{l} ; Q_{1}=P_{l}$, if $P_{l}$ is homogeneous; and, $Q_{l} \mathscr{S} \cap B=p_{\jmath}$. Using this, it will now be shown that $Q_{t}=Q_{i+1} \subset Q_{t+2} \subset \cdots \subset Q_{d}$. As just noted, $Q_{t}=p_{t} \mathscr{S} \cap \mathscr{R}=P_{t}$ and $Q_{t+1}=p_{t+1} \mathscr{P} \cap \mathscr{R} \subset P_{t+1}$ (since, by hypothesis, $P_{\imath}$ is homogeneous and $P_{t+1}$ isn't), so $P_{\imath}=Q_{\imath}=Q_{i+1}$, since height $P_{t+1} / P_{\imath}=1$. Also, for $k=$ $1, \cdots, d-i-1, Q_{i+k} \subset P_{i+k} \subset P_{i+k+1}$, since $P_{i+k}$ isn't homogeneous. Now, since it is well known that a chain of three distinct prime ideals in $B[t b, 1 / t b]$ can't all lie over the same prime ideal in $B, Q_{i+k} \mathscr{S} \cap B=$ $p_{t+k}=P_{t+k} \mathscr{S} \cap B \subset P_{t+k+1} \mathscr{S} \cap B=p_{t+k+1}=Q_{t+k+1} \mathscr{S} \cap B$. Therefore, it follows that $Q_{t}=Q_{i+1} \subset Q_{i+2} \subset \cdots \subset Q_{d}$. Finally, $Q_{d}$ is homogeneous and depth $Q_{d} \mathscr{S}=$ depth $p_{d} \mathscr{S} \geqq 1$, so there exists a maximal homogeneous prime ideal $\mathcal{M}$ in $\mathscr{R}$ such that $Q_{d} \subset \mathcal{M}$. Therefore $P=P_{0} \subset \cdots \subset P_{t}=$ $Q_{t+1} \subset Q_{t+2} \subset \cdots \subset Q_{d} \subset \mathcal{M}$, so height $\mathcal{M} / P \geqq d=\operatorname{depth} P$, hence height $M / P=\operatorname{depth} P$.

Finally, if $b$ is a zero divisor, then let $K=\cup\left\{(0): b^{\prime} A ; s \geqq 1\right\}$, and let $K^{*}=K A[t, u] \cap \mathscr{R}$. (Note that $b$ isn't nilpotent, since $t b \notin N$, so $K \neq A$.) Then $K^{*} \subseteq P$, for if $z^{*}$ is a prime divisor of zero in $\mathscr{R}$ such that $z^{*} \subseteq P$, then $t b \notin z^{*}$ so $b \notin z=z^{*} \cap A$ (since $z^{*}=z A[t, u] \cap \mathscr{R}$ (2.2.2)), hence $K \subseteq z$, so $K^{*} \subseteq z^{*} \subseteq P$. Therefore depth $P=\operatorname{depth}$ $P / K^{*}, \mathscr{R} / K^{*} \cong \mathscr{R}(A / K,(I+K) / K)(2.23), t b+K^{*} \notin P / K^{*}$, and $b+K$ is regular in $A / K$, so the conclusion follows from the case $b$ is regular in $A$.

The following corollary is an important special case of (3.7).

(3.8) Corollary. With $A, I$, and $\mathscr{R}$ as in (3.7), if altitude $A<\infty$ and $p$ is a prime divisor of $u \mathscr{R}$, then there exists a maximal homogeneous prime ideal $\mathcal{M}$ in $\mathscr{R}$ such that $p \subseteq \mathcal{M}$ and depth $p=$ height $\mathcal{M} / p$.

Proof. This is clear by (3.7), since each prime divisor of $u \mathscr{R}$ is homogeneous.

(8.5) and the comments following (8.1) and (9.1) contain other results which are related to the results in this section. 
4. Prime divisors of zero in $\mathscr{F}(R, I)$. The prime divisors of $u \mathscr{R}(R, I)$ are considered in this section, where $I$ is an arbitrary ideal contained in the Jacobson radical of a semi-local ring $R$. It is shown that there is a close relationship between these prime divisors and the prime divisors of $u \mathscr{R}\left(R^{*}, I R^{*}\right)$ (2.3.2). (In $\S 5$, the case where $I$ is open will be considered, and the results in the present section will be considerably sharpened in this case.) We begin with the following result which is a slight generalization and sharpening of [10, Lemma 3.2]. (It should be noted that the rings $\mathscr{R}(R, I)$ are special cases of the rings $A$ in (4.1).) The lemma will be of frequent use in the remainder of this paper.

(4.1) Lemma. Let $R$ be a semi-local ring, and let $A=$ $R\left[X_{1}, \cdots, X_{k}, y_{1}, \cdots, y_{d}\right]$, where the $X_{\imath}$ are algebraically independent over $R$ and the $y_{1}$ are in the total quotient ring of $R\left[X_{1}, \cdots, X_{k}\right]$. Let $C=R^{*}\left[X_{1}, \cdots, X_{k}, y_{1}, \cdots, y_{d}\right](2.3 .2)$, so $A \subseteq C$. Then there is a one -toone correspondence between the prime ideals $P$ in $A$ such that $P \cap R$ is a maximal ideal and the prime ideals $P^{*}$ in $C$ such that $P^{*} \cap R^{*}$ is maximal given by $P=P^{*} \cap A$ and $P^{*}=P C$. Moreover, if $P$ and $P^{*}$ correspond, then $A_{p}$ is a dense subspace of $C_{P^{*}}$ (so height $P=$ height $P^{*}$ ), depth $P=$ depth $P^{*}$, and $P$ is a prime divisor of an ideal $B$ in $A$ if and only if $P^{*}$ is a prime divisor of $B C$.

Proof. The one-to-one correspondence and the fact that $A_{p}$ is a dense subspace of $C_{P^{*}}$ both follow from [10, Lemma 3.2] (which proves these two statements hold when $R$ is local). Further, depth $P=$ depth $P^{*}$, since $C / P^{*}=A / P$ (since $P$ and $P^{*}$ lie over corresponding maximal ideals in $R$ and $R^{*}$ ). Finally, since $A_{p}$ is a dense subspace of $C_{P^{*}}, P$ is a prime divisor of $B$ if and only if $P^{*}$ is a prime divisor of $B C$, by [5, (18.11)].

The next result shows that the prime divisors of $u \mathscr{R}\left(R^{*}, I R^{*}\right)$ give considerable information on the prime divisors of $u \mathscr{R}(R, I)$, and vice versa.

(4.2) Proposition. Let $I$ be an ideal in a semi-local ring $R$ such that $I \subseteq J$, the Jacobson radical of $R$, let $\mathscr{R}=\mathscr{R}(R, I)$, and let $\mathscr{R}^{0}=$ $\mathscr{R}\left(R^{*}, I R^{*}\right)$ (2.3.2). Then the following statements hold:

(4.2.1) If $p^{0}$ is a prime divisor of $u \mathscr{R}^{0}$, then $p=p^{0} \cap \mathscr{R}$ is a prime divisor of $u \mathscr{R}, p^{0}$ is a prime divisor of $p \mathscr{R}^{0}$, and $\mathscr{R}_{p}$ is a subspace of $\mathscr{R}_{p^{0}}{ }^{0}$. (In particular, this holds if $z$ is a prime divisor of zero in $R^{*}$ and $p^{0}$ is any prime ideal in $\mathscr{R}^{0}$ which is minimal with respect to containing $\left(z R^{*}[t, u] \cap \mathscr{R}^{0}, u\right) \mathscr{R}^{0}$, and then depth $p^{0}=$ depth $z$.) 
(4.2.2) If $p$ is a prime divisor of $u \mathscr{R}$, then, for each prime divisor $p^{0}$ of $p \mathscr{R}^{0}, p^{0}$ is a prime divisor of $u \mathscr{R}^{0}$ and $\mathscr{R}_{p}$ is a subspace of $\mathscr{R}_{p^{0}}$.

(4.2.3) With $p$ as in (4.2.2), if $p^{0}$ is a minimal prime divisor of $p \mathscr{R}^{0}$, then height $p^{0}=$ height $p$ and depth $p^{0} \leqq$ depth $p$. Moreover, there exists a minimal prime divisor $P$ of $p \mathscr{R}^{0}$ such that depth $P=$ depth $p$.

Proof. (4.2.1) Let $\mathcal{M}^{0}$ be a maximal homogeneous ideal in $\mathscr{R}^{0}$ such that $p^{0} \subseteq \mathcal{M}^{0}$, and let $\mathcal{M}=\mathcal{M}^{0} \cap \mathscr{R}$. Then $\mathscr{R}_{\mathcal{M}}$ is a dense subspace of $\mathscr{R}_{\mathcal{M}^{0}}^{0}$ (by (4.1), since $\mathcal{M}^{0} \cap R^{*}$ is maximal (2.2.1)). Therefore, since $u \mathscr{R}_{\mathcal{M}^{0}}^{0}$ is an extended ideal from $\mathscr{R}_{\mathscr{M}}, p=p^{0} \cap \mathscr{R}$ is a prime divisor of $u \mathscr{R}$ and $p^{0}$ is a prime divisor of $p \mathscr{R}^{0}$, by $[\mathbf{5},(18.11)]$, hence $\mathscr{R}_{p}$ is a subspace of $\mathscr{R}_{p^{0}}^{0}[\mathbf{5}$, (19.2) (3)].

For the parenthetical statement, let $z^{*}=z R^{*}[t, u] \cap \mathscr{R}^{0}$, where $z$ is a prime divisor of zero in $R^{*}$, so $z^{*}$ is a prime divisor of zero in $\mathscr{R}^{0}$ (2.2.2). Let $p^{0}$ be a minimal prime divisor of $\left(z^{*}, u\right) \mathscr{R}^{0}$. (Note that $\left(z^{*}, u\right) \mathscr{R}^{0} \neq \mathscr{R}^{0}$, since $I \subseteq J$.) Then $p^{0}$ is a prime divisor of $u \mathscr{R}^{0}$, by [21, Lemma 1, p. 394] applied to $\mathscr{R}_{p^{\prime \prime}}^{0}$. Also, since $\mathscr{R}^{0} / z^{*} \cong$ $\mathscr{R}\left(R^{*} / z,\left(I R^{*}+z\right) / z\right)(2.2 .3)$ and $R^{*} / z$ is a complete local domain, $\mathscr{R}^{\prime \prime} / z^{*}$ is catenary [9, Corollary 3.7] and has exactly one maximal homogeneous prime ideal $N=\mathcal{M}^{0} / z^{*}$ (2.2.1). (Hence depth $p^{0} / z^{*}=$ height $N /\left(p^{0} / z^{*}\right)$, by (3.8). Therefore, since height $p^{0} / z^{*}=1$ and height $N=$ depth $z+1$ (2.2.4), depth $p^{0}=$ depth $p^{0} / z^{*}=$ height $N$ - height $p^{0} / z^{*}=\operatorname{depth} z$.

(4.2.2) Let $p$ be a prime divisor of $u \mathscr{R}$ and let $p^{0}$ be a prime divisor of $p \mathscr{R}^{0}$. Then $p^{0}$ is homogeneous, since $p$ is, so there exists a maximal homogeneous ideal $\mathcal{M}^{0}$ in $\mathscr{R}^{0}$ such that $p^{0} \subseteq \mathcal{M}^{0}$. Then, since $\mathcal{M}^{0} \cap \mathscr{R}^{*}$ is a maximal ideal (2.2.1), $\mathscr{R}_{\mathcal{M}}$ is a dense subspace of $\mathscr{R}_{\mu^{\prime \prime}}^{0}(4.1)$, where $\mathcal{M}=\mathscr{M}^{0} \cap \mathscr{R}$. Therefore, since $p$ is a prime divisor of $u \mathscr{R}, p^{0}$ is a prime divisor of $u \mathscr{R}^{0}$ and $\mathscr{R}_{p}$ is a subspace of $\mathscr{R}_{p^{0}}^{0}$, by $[5,(18.11)]$ and $[5,(19.2)(3)]$.

(4.2.3) Let $p^{0}$ be a minimal prime divisor of $p \mathscr{R}^{0}$. Then, with $\mathscr{M}^{0}$ and $\mathcal{M}$ as in the proof of (4.2.2), $\mathscr{R}_{\mathfrak{M}}$ is a dense subspace of $\mathscr{R}_{\mathcal{M}^{0}}^{0}$, so height $p^{0}=$ height $p[5,(22.9)]$. Also, it was shown in the proof of $(4.2 .1)$ that depth $p^{0}=$ height $\mathcal{M}^{0} / p^{0}$. Thus, since $\mathscr{R}_{\mathcal{M}} / p \mathscr{R}_{\mathcal{M}}$ is a dense subspace of

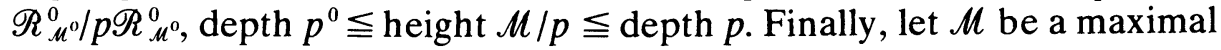
homogeneous prime ideal in $\mathscr{R}$ such that $p \subseteq \mathcal{M}$ and height $\mathcal{M} / p=\operatorname{depth}$ $p$ (3.8). Then $\mathscr{R}_{\mathcal{M}} / p \mathscr{R}_{\mathcal{M}}$ is a dense subspace of $\mathscr{R}_{M \mathscr{R}^{n}}^{0} / p \mathscr{R}^{0}{ }^{0} \mathscr{R}^{n}$, by (4.1), so there exists a minimal prime divisor $P$ of $p \mathscr{R}^{0}$ such that $P \subseteq M \mathscr{R}^{0}$ and height $M / p=$ height $M \mathscr{R}^{0} / P \leqq$ depth $P$. Therefore depth $p \leqq \operatorname{depth} P$, and, as shown above, depth $P \leqq \operatorname{depth} p$. 
(4.3) shows that, because of (4.2), quite a lot of information relating the depths of the prime divisors of $u \mathscr{R}$ and of $u \mathscr{R}^{0}$ can be obtained. (It should be noted that (4.3) greatly extends part of [10, Proposition 3.9] where it was shown that the first set in (4.3) is contained in the third set when $I$ is an open ideal in a local ring $R$.)

(4.3) Corollary. With $R, I, \mathscr{R}$, and $\mathscr{R}^{0}$ as in (4.2), \{depth $p$; $p$ is a minimal prime divisor of $u \mathscr{R}\} \subseteq\left\{\right.$ depth $p^{0} ; p^{0}$ is a minimal prime divisor of $\left.u \mathscr{R}^{0}\right\} \subseteq\left\{\right.$ depth $z ; z$ is a minimal prime ideal in $\left.R^{*}\right\}$ and depth $P ; P$ is a prime divisor of $u \Re\} \cup\left\{\right.$ depth $z ; z$ is a prime divisor of zero in $\left.R^{*}\right\} \subseteq$ $\left\{\right.$ depth $P^{0} ; P^{0}$ is a prime divisor of $\left.u \mathscr{R}^{0}\right\}$.

Proof. Let the sets be, in order of appearance, $S_{i}(i=1, \cdots, 6)$. Then $S_{1} \subseteq S_{2}$ and $S_{4} \subseteq S_{6}$, by (4.2.3). Also, $S_{5} \subseteq S_{6}$, by (4.2.1), since $\left(z R^{*}[t, u] \cap \mathscr{R}^{0}, u\right) \mathscr{R}^{0} \neq \mathscr{R}^{0}$, for all prime divisors $z$ of zero in $R^{*}$ (since $I \subseteq J)$. Finally, if $p^{0}$ is a minimal prime divisor of $u \mathscr{R}^{0}$, then there exists a minimal prime ideal $z$ in $R^{*}$ such that $z^{*}=z R^{*}[t, u] \cap \mathscr{R}^{0} \subset p^{0}(2.2 .2)$, and then depth $p^{0}=$ depth $z$ (4.2.1), so $S_{2} \subseteq S_{3}$.

The following remark, part of which is needed for the proof of (4.5), generalizes parts of (4.2). The proof of the remark is essentially the same as that given for (4.2), so it will be omitted.

(4.4) Remark. With the notation of (4.2), let $H$ be a homogeneous ideal in $\mathscr{R}$. Then the following statements hold:

(4.4.1) If $P^{0}$ is a prime divisor of $H \mathscr{R}^{0}$, then $P=P^{0} \cap \mathscr{R}$ is a prime divisor of $H, P^{0}$ is a prime divisor of $P \mathscr{R}^{0}$, and $\mathscr{R}_{P}$ is a subspace of $\mathscr{R}_{P^{0}}^{0}$.

(4.4.2) If $P$ is a prime divisor of $H$ and $P^{0}$ is a prime divisor of $P \mathscr{R}^{0}$, then $P^{0}$ is a prime divisor of $H \mathscr{R}^{0}$ and $\mathscr{R}_{P}$ is a subspace of $\mathscr{R}_{P^{0}}^{0}$.

(4.4.3) With $P$ as in (4.4.2), if $P^{0}$ is a minimal prime divisor of $P \mathscr{R}^{0}$, then height $P^{0}=$ height $P$ and depth $P^{0} \leqq$ depth $P$. Moreover, there exists a minimal prime divisor $P^{0}$ of $P \mathscr{R}^{0}$ such that depth $P^{0}=\operatorname{depth} P$.

The following proposition is considerably more general than is actually needed when considering prime divisors of $u \mathscr{R}^{\prime}$. However, the more general result is of importance for other considerations, and its proof isn't appreciably more difficult than when just considering prime divisors of $u \mathscr{R}^{\prime}$. For these reasons, it was decided to prove this more general version of the result.

(4.5) Proposition. With $R, I, \mathscr{R}$, and $\mathscr{R}^{0}$ as in (4.2), let $P^{\prime}$ be a homogeneous prime ideal in $\mathscr{R}^{\prime}$. Then the following statements hold: 
(4.5.1) There exists a prime ideal $P^{0 \prime}$ in $\mathscr{R}^{0 \prime}$ such that $P^{0 \prime} \cap \mathscr{R}^{\prime}=P^{\prime}$, height $P^{0 \prime}=$ height $P^{\prime}$, and depth $P^{0 \prime}=$ depth $P^{\prime}$.

(4.5.2) Assume that height $P^{\prime}=1$ and $P^{\prime}$ contains a regular element, and let $T$ be the total quotient ring of $R^{*}$. If $z$ is the minimal prime ideal in $R^{*}$ such that $z T[t, u] \cap \mathscr{R}^{0 \prime} \subset P^{\prime \prime}$ (with $P^{0 \prime}$ as in (4.5.1)), then depth $P^{\prime}=\operatorname{depth} P^{\prime \prime \prime}=$ depth $z$.

Proof. (4.5.1) Let $c \in P^{\prime}$ such that $c$ isn't in any other prime ideal in $\mathscr{R}^{\prime}$ which lies over $p=P^{\prime} \cap \mathscr{R}$, and let $P=P^{\prime} \cap \mathscr{R}[c]$, so height $P=$ height $P^{\prime}$ and depth $P=\operatorname{depth} P^{\prime}$. Now $p$ is homogeneous, since $P$ is, so there exists a maximal homogeneous prime ideal $\mathcal{M}$ in $\mathscr{R}$ such that depth $P=\operatorname{depth} p=$ height $\mathcal{M} / p$ (3.8). Also, $\mathcal{M} \cap \mathscr{R}$ is a maximal ideal (2.2.1). Therefore there exists a maximal ideal $\mathcal{N}$ in $\mathscr{R}[c]$ such that $\mathcal{N} \cap \mathscr{R}=\mathcal{M}$ (so $\mathcal{N} \cap R$ is maximal) and depth $P=$ height $\mathcal{N} / P$. Then $\mathcal{N}^{0}=\mathcal{N} \mathscr{R}^{0}[c]$ is a maximal ideal and $\mathscr{R}[c]_{\mathcal{N}}$ is a dense subspace of $\mathscr{R}^{0}[c]_{\mathcal{N}^{\prime \prime}}$ (4.1). Therefore there exists a minimal prime divisor $Q$ of $P \mathscr{R}^{\circ}[c]$ such that $Q \cap \mathscr{R}[c]=P$, height $Q=$ height $P$, and depth $Q \geqq$ height $\mathcal{N}^{\circ} / Q=$ height $\mathcal{N} / P=$ depth $P$. On the other hand, $\left(Q \cap \mathscr{R}^{0}\right) \cap \mathscr{R}=P \cap \mathscr{R}=p$, so $Q \cap \mathscr{R}^{0}$ contains a minimal prime divisor $p^{0}$ of $p \mathscr{R}^{0}$, hence depth $Q=$ depth $Q \cap \mathscr{R}^{0} \leqq$ depth $p^{0} \leqq(4.4 .3)$ depth $p=\operatorname{depth} P$. Thus depth $Q=$ depth $P$. Now there exists a prime ideal $P^{0 \prime}$ in $\mathscr{R}^{0 \prime}$ such that $P^{0 \prime} \cap \mathscr{R}^{0}[c]=Q$ and height $P^{0 \prime}=$ height $Q$ (and depth $P^{0 \prime}=\operatorname{depth} Q$ ), and then $P^{0 \prime} \cap \mathscr{R}^{\prime}=P^{\prime}$, since $P^{0 \prime} \cap \mathscr{R}[c]=P$.

(4.5.2) By assumption and (4.5.1), height $P^{0 \prime}=1$ and $P^{0 \prime}$ contains a regular element. Therefore, by (2.4.1), there exists a unique minimal prime ideal $z^{* \prime}$ in $\mathscr{R}^{0 \prime}$ such that $z^{*^{\prime}} \subset P^{0 \prime}$, and then $z^{* \prime}=z T[t, u] \cap \mathscr{R}^{\prime \prime}$, where $z=z^{* \prime} \cap R^{*}$ is a minimal prime ideal. Let $z^{*}=z^{* \prime} \cap \mathscr{R}^{0}$ and $p^{0}=P^{0 \prime} \cap \mathscr{R}^{0}$, so $\mathscr{R}^{0} / z^{*}$ satisfies the chain condition for prime ideals (by [9, Corollary 3.7], since $R^{*} / z$ does [5, (34.4)]), hence height $p^{0} / z^{*}=1$, since height $P^{0 \prime} / z^{* \prime}=1$ and $\left(P^{0 \prime} / z^{* \prime}\right) \cap\left(\mathscr{R}^{0} / z^{*}\right)=p^{0} / z^{*}$. Also, as in the second paragraph of the proof of (4.2.1), depth $p^{0}=\operatorname{depth} z$, so depth $P^{0 \prime}=$ depth $p^{0}=\operatorname{depth} z$.

Concerning (4.5), it would be interesting to know to what extent similar statements hold concerning a given homogeneous prime ideal $P^{0 \prime}$ in $\mathscr{R}^{0 \prime}$ and $P^{\prime}=P^{0 \prime} \cap \mathscr{R}^{\prime}$. For example, I am unable to show (or to disprove) that if $P^{0 \prime}$ is a (minimal) prime divisor of $u \mathscr{R}^{0 \prime}$, then $P^{0 \prime} \cap \mathscr{R}^{\prime}$ is a (minimal) prime divisor of $u \mathscr{R}^{\prime}$. (This does hold if $I$ is open, as will be shown in (5.1.3) below.)

The next result specializes (4.5) to the prime divisors of $u \mathscr{R}^{\prime}$ and of $u \mathscr{R}^{0 \prime}$. 
(4.6) Corollary. With $R, I, \mathscr{R}$ and $\mathscr{R}^{0}$ as in (4.2), $\left\{\right.$ depth $p^{\prime} ; p^{\prime}$ is a prime divisor of $\left.u \mathscr{R}^{\prime}\right\} \subseteq\left\{\right.$ depth $p^{0 \prime} ; p^{0 \prime}$ is a prime divisor of $\left.u \mathscr{R}^{0 \prime}\right\}=\{$ depth $z ; z$ is a minimal prime ideal in $\left.R^{*}\right\}$.

Proof. Let $p^{\prime}$ be a prime divisor of $u \mathscr{R}^{\prime}$, so height $p^{\prime}=1$ (2.4.1). Therefore, by (4.5.2), there exists a prime divisor $p^{0 \prime}$ of $u \mathscr{R}^{0 \prime}$ such that $p^{0 \prime} \cap \mathscr{R}^{\prime}=p^{\prime}$ and depth $p^{0 \prime}=$ depth $p^{\prime}$. Therefore the first containment holds.

Now let $p^{0 \prime}$ be a (minimal) prime divisor of $u \mathscr{R}^{0 \prime}$. Then depth $p^{0 \prime}=$ depth $z$, for some minimal prime ideal $z$ in $R^{*}(4.5 .2)$, so the second containment holds.

Finally, if $z$ is a minimal prime ideal in $R^{*}$ and $z^{*}=z T[t, u] \cap \mathscr{R}^{0 \prime}$, then there exists a minimal prime divisor $p^{0 \prime}$ of $u \mathscr{R}^{0 \prime}$ such that $z^{*} \subset p^{0 \prime}$ [12, Corollary 2.23], and then depth $z=$ depth $p^{0 \prime}$, as in the proof of (4.5.2). Therefore, the last two sets are equal.

The last corollary to (4.2) shows that, in the semi-local case, certain additional prime divisors of $u \mathscr{R}\left(R, I^{n}\right)$ (besides those considered in $\S 3$ ) give information on the prime divisors of $u \mathscr{R}(R, I)$.

(4.7) Corollary. Let $R, I, \mathscr{R}$, and $\mathscr{R}^{0}$ be as in (4.2). Fix a positive integer $n$ and let $\mathscr{P}=\mathscr{R}\left(R, I^{n}\right)$ and $\mathscr{P}^{0}=\mathscr{R}\left(R^{*}, I^{n} R^{*}\right)$. Then, if $q^{0 \prime}$ is a prime divisor of $u \mathscr{P}^{0 \prime}$, then there exists a prime divisor $p$ of $u \mathscr{R}$ such that depth $p=$ depth $q^{0 \prime} \cap \mathscr{P}$.

Proof. Let $q^{0 \prime}$ be a prime divisor of $u \mathscr{P}^{0 \prime}$ and $q=q^{0 \prime} \cap \mathscr{P}$, so $q$ is a prime divisor of $u \mathscr{P}$, by (2.4.2) and (4.2.1) applied to $\mathscr{P}$ and $\mathscr{P}^{0}$. Let $\mathscr{A}=R\left[t^{n} I^{n}, u^{n}\right]$ and $\mathscr{A}^{0}=R^{*}\left[t^{n}\left(I^{n} R^{*}\right), u^{n}\right]$, so $\mathscr{A}^{0 \prime} \cong \mathscr{P}^{0 \prime}$ and $\mathscr{A} \cong \mathscr{P}$, hence $u^{n} \mathscr{A}^{0 \prime}$ has a prime divisor $m^{0 \prime}$ which corresponds to $q^{0 \prime}$ under the isomorphism, so $m=m^{0 \prime} \cap \mathscr{A}$ is a prime divisor of $u^{n} \mathscr{A}$ such that depth $m=$ depth $q$. Now $\mathscr{R}^{0 \prime}$ contains and is integral over $\mathscr{A}^{0 \prime}$, so there exists a prime divisor $p^{0 \prime}$ of $u \mathscr{R}^{0 \prime}$ such that $p^{0 \prime} \cap \mathscr{A}^{0 \prime}=m^{0 \prime}$ (since height $m^{0 \prime}=1$ (2.4.1)). Then $p=p^{0 \prime} \cap \mathscr{R}$ is a prime divisor of $u \mathscr{R}$ such that $p \cap \mathscr{A}=$ $m$. Therefore, since $\mathscr{R}$ is integral over $\mathscr{A}$, depth $p=\operatorname{depth} m=\operatorname{depth} q$.

5. Prime divisors of zero in $\mathscr{F}(R, Q)$. In this section, we consider the prime divisors of $u \mathscr{R}(R, Q)$, where $Q$ is an open ideal in a semi-local ring $R$. For this case, it is shown that most of the results in $\S 4$ can be considerably sharpened.

Concerning (5.1), it should be noted that (5.1.1) (respectively, (5.1.2), (5.1.3), (5.1.4)) sharpens (4.2) (respectively, (4.3), (4.6), (3.3) and (4.6)). It should also be noted that part of (5.1.1) is known [1, Proposition 10.22(ii)]. (Note that this result in [1] isn't applicable in $\S 4$ of this 
paper, since the completion in [1] is the $\mathfrak{A}$-adic completion, whereas, in $\S 4, R^{*}$ is the completion of $R$ with respect to its Jacobson radical.) Finally, there is some overlap between (5.1.1) and [13, Theorem 5.9] (which is concerned with an equi-characteristic local ring).

(5.1) Theorem. Let $Q$ be an open ideal in a semi-local ring $R$, let $\mathscr{R}=\mathscr{R}(R, Q)$, and let $\mathscr{R}^{0}=\mathscr{R}\left(R^{*}, I R^{*}\right)$. Then the following statements hold:

(5.1.1) There exists a one-to-one correspondence between the prime divisors $p$ of $u \mathscr{R}$ and the prime divisors $p^{0}$ of $u \mathscr{R}^{0}$ such that $p$ and $p^{0}$ correspond if and only if $p=p^{0} \cap \mathscr{R}$ and $p^{0}=p \mathscr{R}^{0}$, and then $\mathscr{R}_{p}$ is a dense subspace of $\mathscr{R}_{p^{0}}^{0}$ (so height $p=$ height $p^{0}$ ) and depth $p=$ depth $p^{0}$.

(5.1.2) $\{$ depth $p ; p$ is a minimal prime divisor of $u \mathscr{R}\}=\left\{\right.$ depth $p^{0} ; p^{0}$ is a minimal prime divisor of $\left.u \mathscr{R}^{0}\right\} \subseteq\{$ depth $z$; $z$ is a minimal prime ideal in $\left.R^{*}\right\} \subseteq\left\{\right.$ depth $w ; w$ is a prime divisor of zero in $\left.R^{*}\right\} \subseteq\{$ depth $P$; $P$ is a prime divisor of $u \mathscr{R}\}=\left\{\right.$ depth $P^{0} ; P^{0}$ is a prime divisor of $\left.u \mathscr{R}^{0}\right\}$.

(5.1.3) $\quad\left\{p^{0 \prime} \cap \mathscr{R}^{\prime} ; p^{0 \prime}\right.$ is a prime divisor of $\left.u \mathscr{R}^{0 \prime}\right\}=\left\{p^{\prime} ; p^{\prime}\right.$ is a prime divisor of $\left.u \mathscr{R}^{\prime}\right\}$. Moreover, if $p^{0 \prime}$ is the only prime divisor of $u \mathscr{R}^{0 \prime}$ which lies over $p^{\prime}=p^{0 \prime} \cap \mathscr{R}^{\prime}$, then $\left(\mathscr{R}_{p}^{\prime}\right) /\left(\operatorname{Rad} \mathscr{R}_{p^{\prime}}^{\prime}\right)$ is a. dense subspace of $\left(\mathscr{R}_{p^{\prime \prime}}^{0 \prime} /\left(\operatorname{Rad} \mathscr{R}_{p^{\prime \prime}}^{0 \prime}\right)\right.$, and these rings are $D V R^{\prime}$ 's.

(5.1.4) For each $n \geqq 1$, there is a one-to-one correspondence between the prime divisors of $u \mathscr{R}\left(R, Q^{n}\right)^{\prime}$ and the prime divisors of $u \mathscr{R}^{\prime}$, and depth $q^{\prime} ; q^{\prime}$ is a prime divisor of $\left.u \mathscr{R}\left(R, Q^{n}\right)^{\prime}\right\}=\{$ depth $z ; z$ is a minimal prime ideal in $R^{*}$.

Proof. (5.1.1) Since $Q$ is open and $u \mathscr{R} \cap R=Q$, this follows from (4.1).

(5.1.2) Let $S_{i}$ denote, in order, the $i$ th set $(i=1, \cdots, 6)$. Then $S_{1}=S_{2}$ and $S_{5}=S_{6}$, by (5.1.1), and clearly $S_{3} \subseteq S_{4}$. Also, $S_{2} \subseteq S_{3}$ and $S_{4} \subseteq S_{5}$, by (4.3).

(5.1.3) If $p^{\prime}$ is a prime divisor of $u \mathscr{R}^{\prime}$, then height $p^{\prime}=1(2.4 .1)$, so there exists a prime divisor $p^{0 \prime}$ of $u \mathscr{R}^{0 \prime}$ such that $p^{0 \prime} \cap \mathscr{R}^{\prime}=p^{\prime}$, by (4.5.1). On the other hand, if $p^{0 \prime}$ is any prime divisor of $u \mathscr{R}^{0 \prime}$, then height $p^{0 \prime}=1(2.4 .1)$. To see that height $p^{0 \prime} \cap \mathscr{R}^{\prime}=1$, let $n$ be large, let $B=\left(Q^{n}\right)_{a}$, let $\mathscr{B}=R\left[t^{n} B, u^{n}\right]$, and let $\mathscr{B}^{0}=R^{*}\left[t^{n}\left(B R^{*}\right), u^{n}\right]$. Then $\mathscr{R}^{\prime}$ contains and is integral over $\mathscr{B}$, since $\mathscr{R}$ contains and is integral over $\mathscr{A}=R\left[t^{n} Q^{n}, u^{n}\right]$ and $\mathscr{A} \subseteq \mathscr{B} \subseteq \mathscr{A}^{\prime} \subseteq \mathscr{R}^{\prime}$. Likewise $\mathscr{R}^{0 \prime}$ contains and is 
integral over $\mathscr{B}^{0 \prime}$. Let $q^{0 \prime}=p^{0 \prime} \cap \mathscr{B}^{0 \prime}$ and $q=q^{0 \prime} \cap \mathscr{B}$. Then, by the proof of (3.3), height $q^{0 \prime}=1$. Also, height $q=1$ (by (7.1), since $n$ is large and $\mathscr{B} \cong \mathscr{S}=\mathscr{R}(R, B)$ and $\left.\mathscr{B}^{0 \prime} \cong \mathscr{S}^{0 \prime}=\mathscr{R}\left(R^{*}, B R^{*}\right)^{\prime}\right)$. Therefore, since $\left(p^{0 \prime} \cap \mathscr{R}^{\prime}\right) \cap \mathscr{B}=q$, height $p^{0 \prime} \cap \mathscr{R}^{\prime}=1$.

The proof of the subspace part of (5.1.3) requires some preliminary information (that will be given in $\$ 6$ ). Therefore the proof of (5.1.3) will be completed in the proof of (7.4).

(5.1.4) The one-to-one correspondence is given by (3.3), and the first set is contained in the second, by (3.3) and (4.6). Now let $d$ be an element in the second set and let $p^{0 \prime}$ be a prime divisor of $u \mathscr{R}^{\prime \prime}$ such that depth $p^{\prime \prime \prime}=d$ (4.6). Let $p^{0}=p^{0 \prime} \cap \mathscr{R}^{0}, p=p^{0} \cap \mathscr{R}$, and $p^{\prime}=p^{0 \prime} \cap \mathscr{R}^{\prime}$, so $p^{\prime} \cap \mathscr{R}=p$, hence depth $p^{\prime}=\operatorname{depth} p$ and depth $p^{0 \prime}=\operatorname{depth} p^{0}$, by integral dependence. Also, $p^{0}$ is a prime divisor of $u \mathscr{R}^{0}(2.4 .2)$, so $p$ is a prime divisor of $u \mathscr{R}$ and depth $p=\operatorname{depth} p^{0}=d$, by (5.1.1). Therefore depth $p^{\prime}=\operatorname{depth} p=d$, and $p^{\prime}$ is a prime divisor of $u \mathscr{R}^{\prime}$, by (5.1.3), so the two sets are equal, by (3.3).

In regard to (5.1.2), one is tempted to say that if there exist $g$ prime divisors of zero in $R^{*}$, then $u \mathscr{R}^{0}$ has at least $g$ prime divisors. But this isn't true. For example, if $(R, M)$ is a Macaulay local domain and $Q$ is generated by a system of parameters, then $u \mathscr{R}(R, Q)$ is a primary ideal, by [16, Theorem 2.1(i)], so, by (5.1.1), $u \mathscr{R}\left(R^{*}, Q R^{*}\right)$ is primary, regardless of how many prime divisors of zero there are in $R^{*}$.

In the next remark, (5.2.1) sharpens (4.2.1).

(5.2) REMARK. With the notation of (5.1), the following statements hold:

(5.2.1) If $z$ is a prime divisor of zero in $R^{*}$ and $p^{0}$ is a prime ideal in $\mathscr{R}^{0}$ which is minimal with respect to containing $\left(z R^{*}[t, u] \cap \mathscr{R}^{0}, u\right) \mathscr{R}^{0}$, then $p=p^{0} \cap \mathscr{R}$ is a prime divisor of $u \mathscr{R}, \mathscr{R}_{p}$ is a dense subspace of $\mathscr{R}_{p^{0}}^{0}$, height $p=$ height $p^{0}$, and depth $p=\operatorname{depth} p^{0}=\operatorname{depth} z$.

(5.2.2) For each positive integer $n$, the conclusions of (5.1) and (5.2.1) hold with $\mathscr{R}\left(R, Q^{n}\right)$ and $\mathscr{R}\left(R^{*}, Q^{n} R^{*}\right)$ replacing $\mathscr{R}$ and $\mathscr{R}^{0}$, respectively. Therefore, in particular, \{depth $z ; z$ is a prime divisor of zero in $\left.R^{*}\right\} \subseteq\left\{\right.$ depth $q ; q$ is a prime divisor of $\left.u \mathscr{R}\left(R, Q^{n}\right)\right\}$.

Proof. (5.2.1) $p^{0}$ is a prime divisor of $u \mathscr{R}^{0}$, by (4.2.1), so the conclusions follow from (5.1.1) and (4.2.1).

(5.2.2) is clear, since (5.1) and (5.2.1) hold for all open ideals in all semi-local rings. 
The following corollary is an interesting application of (5.2.1).

(5.3) Corollary. Let $Q$ be an ideal generated by a system of parameters in a local ring $(R, M)$. Then, for all $n \geqq 1, u \mathscr{R}\left(R, Q^{n}\right)$ has only one minimal prime divisor. Moreover, for each prime divisor $z$ of zero in $R^{*}$, there exists a prime divisor $p$ of $u \mathscr{R}\left(R, Q^{n}\right)$ such that depth $p=$ depth $z$ and height $p=$ altitude $R+1-$ depth $p$.

Proof. Let $\mathscr{R}=\mathscr{R}(R, Q), \mathscr{P}=\mathscr{R}\left(R, Q^{n}\right)$, and $\mathscr{A}=R\left[t^{n} Q^{n}, u^{n}\right]$, so $\mathscr{A} \cong \mathscr{P}$ and $\mathscr{R}$ is integral over $\mathscr{A}$. Also, by [10, Lemma 4.3], $N=$ $(M, u) \mathscr{R}$ is the unique minimal prime divisor of $u \mathscr{R}$; height $N=1$; depth $N=$ altitude $R=$ (say) $a$; and, $\mathscr{R} / N$ is a polynomial ring in $a$ indeterminates over $R / M$. Therefore, since $\mathscr{R}$ is integral over $\mathscr{A}, N \cap \mathscr{A}$ is the unique minimal prime divisor of $u^{n} \mathscr{A}$. Also, $\mathscr{R} / N$ is integral over $\mathscr{A} /(N \cap \mathscr{A})$, so, since every maximal chain of prime ideals in $\mathscr{R} / N$ has length $=a$, the same holds for $\mathscr{A} /(N \cap \mathscr{A})[5,(34.3)]$. Further, altitude $\mathscr{A}=$ altitude $\mathscr{R}=a+1$ (2.2.4), so it follows that height $P+$ depth $P=a+1$, for all prime ideals $P$ in $\mathscr{A}$ such that $u^{n} \in P$. Therefore, since $\mathscr{P} \cong \mathscr{A}$, the conclusions follow from (5.2.1) and what has already been shown.

In (5.3), if $Q$ isn't generated by a system of parameters, then the conclusions don't necessarily hold. For example, in [10, Proposition 3.9] it is shown that in every local ring $(R, M)$ there exists an $M$-primary ideal $Q$ such that there is a minimal prime divisor of $u \mathscr{R}(R, Q)$ which has depth $=d$ if and only if there exists a minimal prime ideal $z$ in $R *$ such that depth $z=d$. (So, if $R$ isn't quasi-unmixed, then $u \mathscr{R}(R, Q)$ has a minimal prime divisor $p$ such that height $p+$ depth $p<$ altitude $\mathscr{R}$.)

6. Integrally closed ideals, normal ideals, and $q$ $v$-rings. To prove the theorem in $\$ 7$, quite a lot of information concerning normal ideals and $q-v$-rings is needed. The information on normal ideals is known (but will be reviewed for the convenience of the reader) while the information on $q$ - $v$-rings is new (at least to the author). We begin by recalling the definition of a normal ideal, and then recall some facts on the integral closure of an ideal and on normal ideals.

(6.1) Definition. An ideal $I$ in a $\operatorname{ring} A$ is said to be normal in case every power of $I$ is integrally closed (that is, $\left(I^{n}\right)_{a}=I^{n}$, for all $n \geqq 1$ ).

For example, it is well known that the maximal ideal in a regular local ring is a normal ideal.

(6.2) Remark. The following statements hold for an ideal $I$ in a Noetherian ring $A$ : 
(6.2.1) $Z=\operatorname{Rad} A \subseteq I_{a}$ and $I_{a} / Z=((I+Z) / Z)_{a}$.

(6.2.2) $\left[11\right.$, Lemma 2.4(5)]. $A=A^{\prime}$ if and only if $(b A)_{a}=b A$, for all regular nonunits $b$ in $A$.

(6.2.3) If $b$ is a regular nonunit in $A$ such that $(b A)_{a}=b A$, then $b A$ is a normal ideal.

(6.2.4) $\left[12\right.$, Lemma 2.5]. $\quad\left(u^{n} \mathscr{R}(A, I)\right)_{a} \cap A=\left(I^{n}\right)_{a}$, for all $n \geqq 1$.

(6.2.5) $(u \mathscr{R}(A, I))_{a}=u \mathscr{R}(A, I)$ if and only if $I$ is a normal ideal.

(6.2.6) [18, Theorems 1 and 2]. If $A$ is semi-local and analytically unramified, then $\left(I^{n}\right)_{a}$ is a normal ideal, for all large $n$. $\left(I A^{*}\right)_{a}$.

(6.2.7) If $A$ is semi-local and $I$ is an open ideal, then $I_{a} A^{*}=$

Proof. (6.2.2), (6.2.4), and (6.2.6) are proved in the cited references, and the proof of (6.2.1) is straightforward by working with equations of integral dependence (since $Z$ is nilpotent).

The proof of (6.2.3) is an easy induction argument, using the readily seen facts: if $c \in A$, then $c / b \in A^{\prime}$ if and only if $c \in(b A)_{a}$; and, $b A^{\prime} \cap A=(b A)_{a} . \quad($ See $[12$, Lemma 2.3].)

(6.2.5) Let $\mathscr{R}=\mathscr{R}(A, I)$ and assume that $(u \mathscr{R})_{a}=u \mathscr{R}$. Then, by (6.2.3), $\left(u^{n} \mathscr{R}\right)_{a}=u^{n} \mathscr{R}$, for all $n \geqq 1$, so $I^{n}=u^{n} \mathscr{R} \cap A=\left(u^{n} \mathscr{R}\right)_{a} \cap A=$ $\left(I^{n}\right)_{a}$, by (6.2.4), so $I$ is normal. For the opposite implication, if $I$ is normal, then let $x t^{k}$ be a homogeneous element in $(u \mathscr{R})_{a}$. Then $x t^{k+1} \in \mathscr{R}^{\prime}\left[12\right.$, Lemma 2.3], so $x \in u^{k+1} \mathscr{R}^{\prime} \cap A=\left(I^{k+1}\right)_{a}$, by [12, Lemma 2.3] and (6.2.4). Therefore $x \in I^{k+1}$, by hypothesis, so $x t^{k+1} \in \mathscr{R}$, and so $x t^{k} \in u \mathscr{R}$. Thus, since $u \mathscr{R}$ and $(u \mathscr{R})_{a}$ are homogeneous ideals, it follows that $u \mathscr{R}=(u \mathscr{R})_{a}$.

(6.2.7) It is clear that $I_{a} A^{*} \subseteq\left(I A^{*}\right)_{a}$, so let $x \in\left(I A^{*}\right)_{a} \cap A$. Then there exist $n$ and $c_{j} \in I^{\prime} A^{*}$ such that $x^{n}+c_{1} x^{n-1}+\cdots+c_{n}=0$, so $x^{n} \in I(x, I)^{n-1} A^{*} \cap A=I(x, I)^{n-1} A$, hence $x \in I_{a}$. Therefore $\left(I A^{*}\right)_{a} \cap$ $A=I_{a}=I_{a} A^{*} \cap A$, so, by the one-to-one correspondence between open ideals, $\left(I A^{*}\right)_{a}=I_{a} A^{*}$.

In [11, Definition, p. 213], a quasi-local ring $(S, N)$ was called a quasi- $v$-ring in case altitude $S=1$ and $N=b S$, for some regular element $b \in S$, and a prime ideal $p$ in a ring $A$ was called a quasi- $v$-ideal in case 
$A_{p}$ is a quasi-v-ring. There is a closely related concept which we must work with in $\S 7$, so we now give the definition and make some comments on it.

(6.3) Definition. Let $A$ be a ring and let $p$ be a prime ideal in A. $A$ is a $q-v$-ring in case $A$ is local (Noetherian), altitude $A=1$, $(0): M=(0)$, and $A /(\operatorname{Rad} A)$ is a DVR. $\quad p$ is a $q-v$-ideal in case $A_{p}$ is a $q$ - $v$-ring.

(The reason for the very similar terminology in the preceeding definition and that given in [11] is that the two concepts are quite closely related. Of the two concepts, only $q$ - $v$-rings will be considered in the remainder of this paper.)

(6.4) Remark. The following statements hold for a ring $A$ :

(6.4.1) If $A$ is a $q$ - $v$-ring with maximal ideal $P$, then $\operatorname{Rad} A$ is prime and is the unique prime divisor of zero, and $P=(\operatorname{Rad} A, b) A$, for some regular element $b \in P$.

(6.4.2) If $A$ is Noetherian and $b$ is a regular nonunit in $A$, then $(b A)_{a}=b A$ if and only if $A_{p}$ is a DVR, for all prime divisors $p$ of $b A$ (so each such $p$ is a $q$-v-ideal). If $(b A)_{a}=b A$, then there exists a one-toone correspondence between the prime divisors $p$ of $b A$ and the prime divisors $p^{\prime}$ of $b A^{\prime}$ such that $p$ and $p^{\prime}$ correspond if and only if $A_{p}=A_{p^{\prime}}^{\prime}$.

(6.4.3) If $A$ is a $q$ - $v$-ring, then $A^{*}$ is a $q$ - $v$-ring.

(6.4.4) If the completion of a local ring $A$ is a $q-v$-ring, then $A^{\prime}$ is quasi-local.

Proof. (6.4.1) is clear from the definition.

(6.4.2) The first statement is proved in [11, Corollary 2.11 and Proposition 2.7(6)]. Thus, if $(b A)_{a}=b A$ and $p$ is a prime divisor of $b A$, then $A_{p}$ is a DVR and $A_{A-p}^{\prime}$ is integral over $A_{p}$ and contained in its quotient field, so $A_{p}=A_{A-p}^{\prime}$, hence $p^{\prime}=p A_{p} \cap A^{\prime}$ is a prime divisor of $b A^{\prime}$ such that $A_{p^{\prime}}^{\prime}=A_{p}$. On the other hand, if $p^{\prime}$ is a prime divisor of $b A^{\prime}$, then $p=p^{\prime} \cap A$ is a prime divisor of $b A$ (2.4.2), so by what has already been shown, $A_{p}$ is a DVR and $A_{p}=A_{p^{\prime}}^{\prime}$.

(6.4.3) Let $z$ be the minimal prime ideal in $A$, so $A / z$ is a DVR. Therefore $A^{*} / z A^{*} \cong(A / z)^{*}$ is a $\mathrm{DVR}$, so $z A^{*}=\operatorname{Rad} A^{*}$ (since every minimal prime ideal in $A^{*}$ contains $z A^{*}$ ). Therefore it follows that $A^{*}$ is a $q$ - $v$-ring. 
(6.4.4) Let $z=\operatorname{Rad} A^{*}$, so $z$ is prime and $w=z \cap A=$ $\operatorname{Rad} A$. Also, $\quad A / w \subseteq A^{\prime} /\left(\operatorname{Rad} A^{\prime}\right) \subseteq(A / w)^{\prime}$. Further, $(A / w)^{*} \cong$ $A^{*} / w A^{*}$ has a unique minimal prime ideal, so $(A / w)^{\prime}$ is quasi-local, hence $A^{\prime}$ is quasi-local.

It should be noted that, in (6.4.4), $A$ need not be a $q$ - $v$-ring, even if $A$ is a local domain. In fact, an example of this is given in [5, (E3.2)]. In (7.3) below, additional examples of this will be given.

This section will be closed with the following proposition which is an important specific case of a more general result of $M$. Nagata [4, Proposition 4].

(6.5) Proposition. Let $R$ be a local ring which is a quotient ring of a finitely generated ring over a complete local ring $R_{0}$. Then the following statements hold:

(6.5.1) If $P \in \operatorname{Spec} R$, then $R / P$ is unmixed and analytically unramified.

(6.5.2) If $P^{*} \in \operatorname{Spec} R^{*}$ and $P^{*}$ is a prime divisor of $\left(P^{*} \cap R\right) R^{*}$, then height $P^{*}=$ height $P^{*} \cap R$ and depth $P^{*}=$ depth $P^{*} \cap R$.

Proof. (6.5.1) Let $P$ be a prime ideal in $R$ and let $L=$ $R_{0} /\left(P \cap R_{0}\right)$. Then $R / P$ is pseudo-geometric (by [5, (36.5)], since $L$ is pseudo-geometric $[\mathbf{5},(32.1)])$, so $R / P$ is analytically unramified [5, (36.4)]. Also, $R / P$ is quasi-unmixed (by [9, Corollary 3.7], since $L$, being a complete local domain, is quasi-unmixed), so $R / P$ is quasiunmixed and analytically unramified, hence $R / P$ is unmixed.

(6.5.2) Let $P=P^{*} \cap R$. Then $R / P$ is unmixed (6.5.1), so depth $P^{*}=$ depth $P$ and $P^{*}$ is a minimal prime divisor of $P R^{*}$, hence height $P^{*}=$ height $P[\mathbf{5},(22.9)]$.

7. Prime divisors of zero in $\mathscr{F}\left(R,\left(Q^{n}\right)_{a}\right)$. In this section, the prime divisors of $u \mathscr{R}\left(R,\left(Q^{n}\right)_{a}\right)$ are considered, when $n$ is large. It turns out that, in this case, a number of the previous results in this paper can be sharpened even more than in $\$ 5$.

(7.1) is one of the main results in this paper. It shows that in every semi-local ring $R$ and for every open ideal $B=\left(Q^{n}\right)_{a}$ with $n$ large, the prime divisors of $u \mathscr{R}(R, B)$ have many properties in common with the prime divisors of this ideal in the analytically unramified semi-local ring case. Specifically, if $R$ is analytically unramified and $B$ is as above, then, with $\mathscr{R}=\mathscr{R}(R, B), u \mathscr{R}$ is integrally closed (by (6.2.6) and 
(6.2.5)). Therefore, if $p$ is a prime divisor of $u \mathscr{R}$, then height $p=1$ and $\mathscr{R}_{p}$ is a DVR (6.4.2). (7.1) shows that much the same thing can be said for arbitrary semi-local rings.

(7.1) TheOREM. Let $R$ be a semi-local ring, and let $Q$ be an open ideal in $R$. Fix a large integer $n$, let $B=\left(Q^{n}\right)_{a}$, let $\mathscr{S}=\mathscr{R}(R, B)$, and let $\mathscr{S}^{0}=\mathscr{R}\left(R^{*}, B R^{*}\right)$. Then there exists a one-to-one correspondence be tween the prime divisors $p^{0 \prime}$ of $u \mathscr{Y}^{0 \prime}$ and the minimal prime divisors $p$ of $u \mathscr{S}$ such that $p^{0 \prime}$ and $p$ correspond if and only if $\mathscr{S}_{p}$ is a dense subspace of $\mathscr{S}_{p^{0} \cap \cap \mathscr{S}^{0}}$. Moreover, for each such $p, p^{\mathscr{S}^{0}}=p^{0 \prime} \cap \mathscr{S}^{0}$ is a $q$-v-ideal (6.3) and depth $p=$ depth $p^{0 \prime}$.

Proof. Let $Z=\operatorname{Rad} R^{*}, \quad$ so $\quad R^{*} / Z \quad$ is analytically unramified. Also, it is clear that, for all $n \geqq 1, Z \subseteq\left(Q^{n} R^{*}\right)_{a}=(6.2 .7)$ $\left(Q^{n}\right)_{a} R^{*}$, and $\left(\left(Q R^{*} / Z\right)^{n}\right)_{a}=\left(Q^{n} R^{*} / Z\right)_{a}=(6.2 .1)\left(Q^{n} R^{*}\right)_{a} / Z$. Therefore, for each large $n,\left(Q^{n} R^{*}\right)_{a} / Z$ is normal (6.2.6). Thus, with $\mathscr{P}=$ $\mathscr{R}\left(R^{*} / Z, B R^{*} / Z\right), \quad u \mathscr{P}=(u \mathscr{P})_{a} \quad$ (6.2.5). Also, $\mathscr{P} \cong \mathscr{S}^{0} / Z^{*} \quad$ (2.2.3), where $Z^{*}=Z R^{*}[t, u] \cap \mathscr{S}^{0}$ (so $Z^{*}=\operatorname{Rad} \mathscr{S}^{0}(2.2 .2)$ ).

Now, if $p^{0}$ is a minimal prime divisor of $u \mathscr{S}^{0}$, then $p^{0} / Z^{*}$ is a prime divisor of $u \mathscr{S}^{0} / Z^{*}=\left(u \mathscr{S}^{0} / Z^{*}\right)_{a}$ (since $\left.u \mathscr{P}=(u \mathscr{P})_{a}\right)$, so $\mathscr{S}_{p^{0}}^{0} / Z^{*} \mathscr{S}_{p^{0}} \cong$ $\left(\mathscr{S}^{0} / Z^{*}\right)_{p^{0} / Z^{*}}=$ (say) $V$ is a $\operatorname{DVR}(6.4 .2)$. Therefore $\operatorname{Rad} \mathscr{S}_{p^{0}}^{0^{0}}=Z^{*} \mathscr{S}_{p^{0}}^{0^{\circ}}$ is prime, so, since $u$ is regular, $\mathscr{S}_{p^{\circ}}^{0}$ is a $q-v$-ring (6.3). Further, since $B$ is open, there exists a one-to-one correspondence between the minimal prime divisors $p$ of $u \mathscr{S}$ and the minimal prime divisors $p^{0}$ of $u \mathscr{S}^{0}$ such that $p$ and $p^{0}$ correspond if and only if $p=p^{0} \cap \mathscr{S}$ and $p^{0}=p^{\mathscr{S}^{0}}$, and then depth $p=\operatorname{depth} p^{0}$ and $\mathscr{S}_{p}$ is a dense subspace of $\mathscr{S}_{p^{0}}^{0}(5.1 .1)$. Therefore, for each minimal prime divisor $p$ of $u \mathscr{S}, p \mathscr{S}^{0}$ is a $q-v$-ideal.

To complete the proof, it suffices to show that there exists a one-to-one correspondence between the prime divisors $p^{0 \prime}$ of $u \mathscr{S}^{0 \prime}$ and the minimal prime divisors $p^{0}$ of $u \mathscr{S}^{0}$ given by $p^{0}=p^{0 \prime} \cap \mathscr{S}$ (by the oneto-one correspondence between the minimal prime divisors $p^{0}$ of $u \mathscr{S}^{0}$ and the minimal prime divisors $p$ of $u \mathscr{S}$ noted above). For this, let $Z^{* \prime}=Z T[t, u] \cap \mathscr{S}^{0 \prime}$, where $T$ is the total quotient ring of $R^{*}$, so $Z^{* \prime}=\operatorname{Rad} \mathscr{S}^{0 \prime}$. Then $\mathscr{S}^{0 \prime} / Z^{* \prime}$ is integral over $\mathscr{S}^{0} / Z^{*}$ and is contained in the total quotient ring of $\mathscr{S}^{0} / Z^{*}$. Also, $u \mathscr{S}^{0} / Z^{*}$ is integrally closed (as noted above), so there exists a one-to-one correspondence between the prime divisors of $u \mathscr{S}^{0} / Z^{*}$ and the prime divisors of $u \mathscr{S}^{0 \prime} / Z^{* \prime}$ given by contraction (6.4.2). Further, there exists a one-to-one correspondence between the minimal prime divisors of $u \mathscr{9}^{0}$ and the (minimal) prime divisors of $u \mathscr{S}^{0} / Z^{*}$, and there exists a one-to-one correspondence between the (minimal) prime divisors of $u \mathscr{S}^{0 \prime}$ and the (minimal) prime divisors of $u \mathscr{S}^{0 \prime} / Z^{* \prime}\left(\right.$ since $Z^{*}=\operatorname{Rad} \mathscr{S}^{0}$ and $\left.Z^{* \prime}=\operatorname{Rad} \mathscr{S}^{0 \prime}\right)$. (Each 
prime divisor of $u \mathscr{S}^{0} / Z^{*}$, of $u \mathscr{S}^{0} / Z^{* \prime}$, and of $u \mathscr{S}^{0 \prime}$ is minimal, by (6.4.2) and (2.4.1).) Therefore the desired one-to-one correspondence follows.

We note some facts concerning the proof of (7.1) for future reference.

(7.2) Remark. With the notation of (7.1), the following statements hold:

(7.2.1) $B R^{*} /\left(\operatorname{Rad} R^{*}\right)$ is a normal ideal (equivalently, by (2.2.3) and $(6.2 .5), u \mathscr{S}^{0} /\left(\operatorname{Rad} \mathscr{S}^{0}\right)$ is integrally closed $)$, and it is because of this fact (and not because $B=B_{a}$ ) that the conclusions of (7.1) hold.

(7.2.2) Although $u \mathscr{S}^{0} /\left(\operatorname{Rad} \mathscr{S}^{0}\right)$ is integrally closed, $u \mathscr{S} /(\operatorname{Rad} \mathscr{S})$ may not be integrally closed. (If $u \mathscr{S} /(\operatorname{Rad} \mathscr{S})$ is integrally closed, then $R /(\operatorname{Rad} R)$ is analytically unramified - see (8.2).)

(7.2.3) If there exists an imbedded prime divisor $z$ of zero in $R^{*}$, then $u \mathscr{S}^{0}$ has an imbedded prime divisor (5.2.1), but every prime divisor of $u \mathscr{S}^{0} /\left(\operatorname{Rad} \mathscr{S}^{0}\right)$ has height one (6.4.2).

In (7.1), $p$ need not be a $q-v$-ideal (even though $p \mathscr{S}^{0}$ is and $\mathscr{S}_{p}$ is a dense subspace of $\mathscr{S}_{p \varphi^{\prime \prime}}^{0}$. In fact, the following example shows that over many local domains there exists a locality $L$ such that $L^{*}$ is a $q-v$-ring, but $L$ isn't a $q$ - $v$-ring.

(7.3) Example. Let $(R, M)$ be a local domain such that there exists at least one minimal prime ideal $w$ in $R^{*}$ such that depth $w=$ altitude $R^{*}$ and $w$ isn't the $w$-primary component of zero. Then there exists a locality $(L, P)$ over $R$ such that $L^{*}$ is a $q$-v-ring and $L$ isn't a $q$ - $v$-ring.

Proof. Let $n$ be large, let $B=\left(M^{n}\right)_{a}$, let $\mathscr{S}=\mathscr{R}(R, B)$, and let $\mathscr{S}^{0}=\mathscr{R}\left(R^{*}, B R^{*}\right)$. Let $p^{0}$ be a minimal prime divisor of $\left(u, w^{*}\right) \mathscr{S}^{0}$, where $w^{*}=w R^{*}[t, u] \cap \mathscr{S}^{0}$, let $p=p^{0} \cap \mathscr{S}$, let $L=\mathscr{S}_{p}$, and let $P=$ $p L$. Then height $p^{0}=1$, since depth $w=$ altitude $R^{*}$. Therefore $\mathscr{S}_{p}^{0}$ o is a $q-v$-ring (by the proof of (7.1)), and $L$ is a dense subspace of $\mathscr{S}_{p}^{0}$ (5.1.1), so $L^{*}$ is a $q-v$-ring (6.4.3) and altitude $L=1$. Therefore, by the definition and with $z$ the unique prime divisor of zero in $L^{*}, L^{*} / z$ is a DVR and $P^{*}=(z, b) L^{*}$, for some regular element $b \in P^{*}$. Now $z \neq(0)$, since $z \cap \mathscr{S}_{p^{0}}^{0}=w^{*} \mathscr{Y}_{p^{0}}^{0} \neq(0)$ (since $w$ isn't the $w$-primary component of zero). Suppose that $L$ is a $q-v$-ring. Then $L$ is a DVR, since $L$ is a local domain, hence $L^{*}$ is a DVR; contradiction. Therefore $L$ isn't a $q$ - $v$-ring.

We now give three corollaries to (7.1). 
(7.4) Corollary. With the notation of (7.1), let $\mathscr{D}^{0 \prime}=\left\{p^{0 \prime} ; p^{0 \prime}\right.$ is a prime divisor of $\left.u \mathscr{S}^{0 \prime}\right\}, \mathscr{D}^{0}=\left\{p^{0} ; p^{0}\right.$ is a minimal prime divisor of $\left.u \mathscr{S}^{0}\right\}$, $\mathscr{D}^{\prime}=\left\{p^{\prime} ; p^{\prime}\right.$ is a prime divisor of $\left.u \mathscr{S}^{\prime}\right\}$, and $\mathscr{D}=\{p ; p$ is a minimal prime divisor of $u \mathscr{S}\}$. Then there exist one-to-one correspondences between these sets given by contraction. Moreover, if $p^{0 \prime}, p^{0}, p^{\prime}$, and $p$ correspond, then these ideals have the same depth, $\mathscr{S}_{p}$ is a dense subspace of $\mathscr{Y}_{p^{n}}^{0}$, and $\left(S_{p^{\prime}}^{\prime}\right) /\left(\operatorname{Rad} \mathscr{S}_{p^{\prime}}^{\prime}\right)$ is a dense subspace of $\left(\mathscr{Y}_{p^{\prime}}^{0}\right) /\left(\operatorname{Rad} \mathscr{S}_{p^{\prime \prime}}^{0}\right)=\left(\mathscr{Y}_{p^{\prime \prime}}^{0 \prime}\right) /\left(\operatorname{Rad} \mathscr{Y}_{p^{\prime \prime}}^{0^{\prime \prime}}\right)$ (and these last three rings are DVRs).

Proof. By the last paragraph of the proof of (7.1) (and since $\left.u \mathscr{S}^{0} / Z^{*}=\left(u \mathscr{S}^{0} / Z^{*}\right)_{a}\right)$, there exists a one-to-one correspondence between the $p^{0} \in \mathscr{D}^{0}$ and the $p^{0 \prime} \in \mathscr{D}^{0 \prime}$ such that $\left(\mathscr{S}_{p^{0}}^{0}\right) /\left(\operatorname{Rad} \mathscr{S}_{p^{0}}^{0}\right) \cong\left(\mathscr{S}^{0} / Z^{*}\right)_{p^{0} / Z^{*}}=$

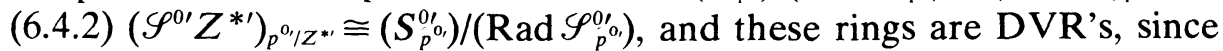
$p^{0}$ is a $q$-v-ideal (7.1). Thus, by this and (7.1), there exists a one-to-one correspondence between the $p \in \mathscr{D}$ and the $p^{0} \in \mathscr{D}^{0}$ such that $\mathscr{S}_{p}$ is a dense subspace of $\mathscr{S}_{p^{\prime}}$. Moreover, the one-to-one correspondence between $\mathscr{D}^{\prime}$ and $\mathscr{D}^{0 \prime}$ follows from the first statement in (5.1.3) and the fact that the $p^{0 \prime} \in \mathscr{D}^{0 \prime}$ contract in $\mathscr{S}$ to distinct height one prime ideals (by what has already been shown), so the $p^{0 \prime}$ contract in $\mathscr{S}^{\prime}$ to distinct height one prime ideals. Thus if $p^{0 \prime}, p^{\prime}, p^{0}$, and $p$ correspond, then $p^{0}=$ $p^{0 \prime} \cap \mathscr{S}^{0}, p^{\prime}=p^{0 \prime} \cap \mathscr{S}^{\prime}$, and $p=p^{0} \cap \mathscr{S}=p^{\prime} \cap \mathscr{S}$, so, by integral dependence and the fact that depth $p^{0}=\operatorname{depth} p$ (5.1.1), it follows that depth $p^{0 \prime}=$ depth $p^{0}=\operatorname{depth} p^{\prime}=\operatorname{depth} p$.

Finally, that $\left(\mathscr{S}_{p^{\prime}}^{\prime}\right) /\left(\operatorname{Rad} \mathscr{S}_{p^{\prime}}^{\prime}\right)$ is a dense subspace of $\left(\mathscr{S}_{p^{\prime},}^{\prime \prime}\right) /\left(\operatorname{Rad} \mathscr{S}_{p^{\prime},}^{\prime \prime}\right)$ follows from the one-to-one correspondence and the more general result stated in (5.1.3), and which will now be proved (using the notation of (5.1)).

Let $p^{0 \prime}$ be a prime divisor of $u \mathscr{R}^{0 \prime}$, let $p^{\prime}=p^{0 \prime} \cap \mathscr{R}^{\prime}$, and assume that $p^{0 \prime}$ is the only prime divisor of $u \mathscr{R}^{0 \prime}$ lying over $p^{\prime}$. Then, to show that $U=\left(\mathscr{R}_{p}^{\prime}\right) /\left(\operatorname{Rad} \mathscr{R}_{p}^{\prime}\right)$ is a dense subspace of $V=\left(\mathscr{R}_{p^{\prime \prime}}^{\prime \prime}\right) /\left(\operatorname{Rad} \mathscr{R}_{p^{\prime \prime}}^{\prime \prime}\right)$, note that $U$ and $V$ are DVR's, by (2.4.1). Also, $U \subseteq V \subseteq V^{*}$, so, by [6, Theorem 7, p. 96], there exists an ideal $K$ in $U^{*}$ such that $U \subseteq U^{*} / K \subseteq$ $V^{*}$, hence $K=(0)$ and $U^{*} \subseteq V^{*}$, since $U, U^{*}$, and $V^{*}$ are DVR's. Let $c \in p^{\prime}$ such that $c$ isn't in any other prime ideal in $\mathscr{R}^{\prime}$ which lies over . $p^{\prime} \cap \mathscr{R}$. Let $\mathscr{A}=\mathscr{R}[c]$ and $\mathscr{A}^{0}=\mathscr{R}^{0}[c]$. Then, with $q=p^{\prime} \cap \mathscr{R}[c]$ and $q^{0}=p^{0 \prime} \cap \mathscr{R}^{0}[c]$, height $q=1, \mathscr{A}_{q}$ is a dense subspace of $\mathscr{A}_{q^{0}}^{0}$, by (4.1), and $\left(\mathscr{A}_{q^{\prime \prime}}^{0}\right)^{\prime}=\mathscr{R}_{p^{\prime \prime}}^{0^{\prime \prime}}$ (since height $q^{0}=$ height $q=1$ and $p^{0 \prime}$ and $p^{\prime}$ are the only prime divisors of $(u)$ lying over $p^{\prime}$ and $q$, respectively). Let $C=\left(\mathscr{A}_{q^{\prime}}^{0}\right) /\left(\operatorname{Rad} \mathscr{A}_{q^{\prime}}^{0}\right)$, so $V=C^{\prime}$ is a finite $C$-algebra (by $[5,(36.4)$ and (32.2)], since $C$ is pseudo-geometric [5, (36.5)]), hence $C^{*} \subseteq C^{* \prime}=$ $V^{*}$. Also, by (6.5.1) and since $\mathscr{A}_{q}$ is a dense subspace of $\mathscr{A}_{q}^{0}$,

$$
C^{*}=\left(\mathscr{A}_{q^{0}}^{0}\right)^{*} /\left(\operatorname{Rad}\left(\mathscr{A}_{q^{o}}^{0}\right)^{*}\right)=\left(\mathscr{A}_{q}\right)^{*} /\left(\operatorname{Rad}\left(\mathscr{A}_{q}\right)^{*}\right) .
$$


Let $L=\mathscr{A}_{q} /\left(\operatorname{Rad} \mathscr{A}_{q}\right)$, so $L \subseteq U \subseteq U^{*}$, hence $L \subseteq L^{*} / I \subseteq U^{*}$, for some ideal $I$ in $L^{*}\left[6\right.$, Theorem 7, p. 96], and $I$ is prime, since $U^{*}$ is a domain. Also, $L^{*}$ is a homomorphic image of $\left(\mathscr{A}_{q}\right)^{*}=\left(\mathscr{A}_{q^{\prime \prime}}^{0}\right)^{*}$ (whose kernel is contained in $\operatorname{Rad}\left(\mathscr{A}_{q^{\prime}}^{0}\right)^{*}$ and $\operatorname{Rad}\left(\mathscr{A}_{q^{\prime}}^{0}\right)^{*}$ is prime, since $C^{*}$ is a domain ('since $\left.C^{*} \subseteq V^{*}\right)$ ). Therefore $C^{*}=L^{*} /\left(\operatorname{Rad} L^{*}\right)$, so, since $C^{*}$ is a domain and altitude $L=1$, it follows that $I=\operatorname{Rad} L^{*}$ and $C^{*}=L^{*} / I \subseteq$ $U^{*}$. Thus $U^{*} \subseteq V^{*}=C^{* \prime} \subseteq U^{* \prime}=U^{*}$, so $U$ is a dense subspace of $V$.

(7.5) REMARK. It follows immediately from (5.1.4) and (7.4) that $\{$ depth $p ; p$ is a minimal prime divisor of $u \mathscr{S}\}=\{$ depth $z ; z$ is a minimal prime ideal in $\left.R^{*}\right\}$.

The next corollary to (7.1) shows that, with $B$ of (7.1) replacing $I$ of (3.1), the first containment in (3.1) becomes an equality. It also shows that equality holds in (3.2).

(7.6) Corollary. Let $R, B$ and $\mathscr{S}$ be as in (7.1), fix $k \geqq 1$, and let $\mathscr{A}=\mathscr{R}\left(R, B^{k}\right)$. Then there exists a one-to-one correspondence between the minimal prime divisors $p$ of $u \mathscr{S}$ and the minimal prim: divisors $q$ of $u \mathscr{A}$, and if $p$ and $q$ correspond, then depth $p=$ depth $q$.

Proof. There exists a one-to-one correspondence between the prime divisors $p^{\prime}$ of $u \mathscr{S}^{\prime}$ and the minimal prime divisors $p$ of $u \mathscr{S}$ given by contraction, by (7.4). Also, $B^{n} R^{*} /\left(\operatorname{Rad} R^{*}\right)$ is a normal ideal (since $B R^{*} /\left(\operatorname{Rad} R^{*}\right)$ is normal (7.2.1)), hence, by (7.1) applied with $B^{n}$ in place of $B$, there exists a one-to-one correspondence between the prime divisors $q^{\prime}$ of $u \mathscr{A}^{\prime}$ and the minimal prime divisors $q$ of $u \mathscr{A}$ given by contraction. Therefore, by the one-to-one correspondence between the prime divisors $p^{\prime}$ of $u \mathscr{S}^{\prime}$ and the prime divisors $q^{\prime}$ of $u \mathscr{A}^{\prime}$ (and since depth $\left.p^{\prime}=\operatorname{depth} q^{\prime}\right)(3.3)$, the conclusion follows.

The last corollary to (7.1) shows that, with $B$ as in (7.1) replacing $I$ in (3.5), the two inequalities in (3.5) become equalities.

(7.7) Corollary. With the notation of (7.1), let $k$ be a positive integer, and let $C$ be an ideal such that $B^{k} \subseteq C \subseteq\left(B^{k}\right)_{a}$. Then there is a one-to-one correspondence between the minimal prime divisors of $(u)$ in the rings $\mathscr{R}\left(R, B^{k}\right), \mathscr{R}(R, C)$, and $\mathscr{R}\left(R, B^{k}\right)^{\prime}$.

Proof. This follows readily from (3.3), (7.1), and (7.6).

An alternate proof to (7.7) is to note that (7.4) is applicable with $\mathscr{R}\left(R, B^{k}\right)$ replacing $\mathscr{S}$ (by $(7.2 .1)$, since $B^{k} R^{*} /\left(\operatorname{Rad} R^{*}\right)$ is a normal ideal). 
8. Some applications. In this section, we give applications of the preceeding results. The first of these is to analytically unramified semi-local rings.

(8.1) Proposition. The following statements are equivalent for a semi-local ring $R$ :

(8.1.1) $\quad R$ is analytically unramified.

(8.1.2) $u \mathscr{R}(R, Q)=(u \mathscr{R}(R, Q))_{a}$, for some open ideal $Q$ in $R$.

(8.1.3) $u \mathscr{R}\left(R^{*}, Q^{*}\right)=\left(u \mathscr{R}\left(R^{*}, Q^{*}\right)\right)_{a}$, for some open ideal $Q^{*}$ in $R^{*}$.

Proof. If (8.1.1) holds, then there exists an open ideal $Q$ in $R$ such that $Q$ is normal $(6.2 .6)$, so, with $\mathscr{R}=\mathscr{R}(R, Q),(u \mathscr{R})_{a}=u \mathscr{R}(6.2 .5)$, hence (8.1.2) holds.

If (8.1.2) holds, then $Q$ is normal (6.2.5), so $Q R^{*}$ is normal, by (6.2.7), hence $u \mathscr{R}\left(R^{*}, Q R^{*}\right)=\left(u \mathscr{R}\left(R^{*}, Q R^{*}\right)\right)_{a}(6.2 .5)$, and so (8.1.3) holds.

Finally, if (8.1.3) holds, then $Q^{*}$ is normal, so $Q=Q^{*} \cap R$ is normal (by (6.2.7), since $Q^{*}=Q R^{*}$ ), so (8.1.1) holds [18, Theorem 2].

If $R$ is analytically unramified in (8.1), then, for all ideals $I$ in $R$ and for all large $n,\left(I^{n}\right)_{a}$ is a normal ideal (6.2.6), so $u \mathscr{R}\left(R,\left(I^{n}\right)_{a}\right)$ is integrally closed (6.2.5).

(8.2) Remark. If there exists an open ideal $Q$ in $R$ such that $(u \mathscr{R})_{a}=u \mathscr{R}+(\operatorname{Rad} \mathscr{R}), \quad$ (equivalently, if $u \mathscr{R} /(\operatorname{Rad} \mathscr{R})$ is integrally closed), where $\mathscr{R}=\mathscr{R}(R, Q)$, then $R /(\operatorname{Rad} R)$ is analytically unramified.

Proof. Let $Z=\operatorname{Rad} R$ and $Z^{*}=Z R[t, u] \cap \mathscr{R}$, so $Z^{*}=\operatorname{Rad} \mathscr{R}$ (2.2.2). Then $R /(\operatorname{Rad} R)$ is analytically unramified, by (8.1) applied to $R / Z$, since $\left(u \mathscr{R}+Z^{*}\right) / Z^{*}=(u \mathscr{R})_{a} / Z^{*}=\left(\left(u \mathscr{R}+Z^{*}\right) / Z^{*}\right)_{a}$ (6.2.1).

Therefore, if $R$ is a local domain which isn't analytically unramified, then, for all open ideals $Q$ in $R$ and with $\mathscr{R}=\mathscr{R}(R, Q)$ and $\mathscr{R}^{0}=$ $\mathscr{R}\left(R^{*}, Q R^{*}\right), u \mathscr{R}$ and $u \mathscr{R}^{0}$ aren't integrally closed, by (8.1). But $u \mathscr{R}^{0} /\left(\operatorname{Rad} \mathscr{R}^{0}\right)$ is integrally closed, by the proof of $(7.1)$, whenever $Q$ is as in (7.1).

It is well known that if $(R, M)$ is a regular local ring, then $M$ has the properties of the ideal $Q$ in the following proposition. 
(8.3) Proposition. Let $(R, M)$ be a local ring which has an $M$ primary ideal $Q$ generated by a system of parameters such that $Q$ is normal. Then $R$ is analytically irreducible and, for all $k \geqq 1, u \Re\left(R, Q^{k}\right)$ is integrally closed and is a primary ideal.

Proof. Let $\mathscr{R}=\mathscr{R}(R, Q)$. Then $u \mathscr{R}=(u \mathscr{R})_{a}(6.2 .5)$, since $Q$ is normal, so every prime divisor of $u \mathscr{R}$ is height one (6.4.2). Also, since $Q$ is generated by a system of parameters, $p=(M, u) \mathscr{R}$ is the only minimal prime divisor of $u \mathscr{R}[\mathbf{1 0}$, Lemma 4.3]. Therefore $u \mathscr{R}$ is $p$-primary. Thus, with $\mathscr{R}^{0}=\mathscr{R}\left(R^{*}, Q R^{*}\right), p^{0}=p \mathscr{R}^{0}$ is the only prime divisor of $u \mathscr{R}^{0}(5.1 .1)$, so every prime divisor of zero in $\mathscr{R}^{0}$ is contained in $p^{0}$, by (5.2.1). Therefore, since $\mathscr{R}_{p}$ is a DVR (6.4.2) and $\mathscr{R}_{p}$ is a dense subspace of $\mathscr{R}_{p^{0}}^{0}(5.1 .1),(0)$ is prime in $\mathscr{R}^{0}$. Therefore $(0)$ is prime in $R^{*}$ (2.2.2), so $R$ is analytically irreducible.

For $k>1, Q^{k}$ is normal, so $u \mathscr{R}\left(R, Q^{k}\right)$ is integrally closed (6.2.5) (so each of its prime divisors has height one) and has only one minimal prime divisor (5.3). Therefore $u \mathscr{R}\left(R, Q^{k}\right)$ is a primary ideal.

The next application concerns quasi-unmixed semi-local rings. To simplify the statement of (8.4), it will be said that an ideal $I$ in a ring $A$ is pure depth $d$ in case every prime divisor of $I$ has depth $=d$.

(8.4) Proposition. Let $R$ be a semi-local ring, and let altitude $R=d$. Then the following statements are equivalent:

(8.4.1) $R$ is quasi-unmixed.

(8.4.2) For every open ideal $Q$ in $R,(u \mathscr{R}(R, Q))_{a}$ is pure depth $d$.

(8.4.3) There exists an open ideal $Q$ in $R$ and a large integer $n$ such that every minimal prime divisor of $u \mathscr{S}$ has depth $=d$, where $\mathscr{S}=$ $\mathscr{R}\left(R,\left(Q^{n}\right)_{a}\right)$.

(8.4.4) There exists an open ideal $Q$ in $R$ such that $u \mathscr{R}(R, Q)^{\prime}$ is pure depth $d$.

Proof. (8.4.4) $\Leftrightarrow$ (8.4.1), by (5.1.4), and (8.4.1) $\Leftrightarrow$ (8.4.3), by (7.5).

If (8.4.1) holds, then let $Q$ be an open ideal in $R$ and let $\mathscr{R}=\mathscr{R}(R, Q)$. Then, since $(u \mathscr{R})_{a}=u \mathscr{R}^{\prime} \cap \mathscr{R}$, the prime divisors of $(u \mathscr{R})_{a}$ are among the ideals $p^{\prime} \cap \mathscr{R}$, where $p^{\prime}$ is a prime divisor of $u \mathscr{R}^{\prime}$. Therefore, since all prime divisors of $u \mathscr{R}^{\prime}$ have depth $=d$, by hypothesis and (5.1.4), $(u \mathscr{R})_{a}$ is pure depth $d$.

Finally, assume that (8.4.2) holds and let $B$ and $\mathscr{S}$ be as in $\S 7$, so there exists a one-to-one correspondence between the prime divisors of 
$u \mathscr{S}^{\prime}$ and the minimal prime divisors of $u \mathscr{S}$ (7.4). Therefore, if $p^{\prime}$ is a prime divisor of $u \mathscr{S}^{\prime}$, then height $p^{\prime} \cap \mathscr{S}=1$, so $p^{\prime} \cap \mathscr{S}$ is a prime divisor of $(u \mathscr{S})_{a}$, hence depth $p^{\prime}=\operatorname{depth} p^{\prime} \cap \mathscr{S}=d$, by hypothesis, hence (8.4.4) holds.

Concerning (8.4), the equivalence of (8.4.1) and (8.4.2) for the local domain case was given in [19, Corollary 4.17].

(8.5) Remark. If $R$ is quasi-unmixed and altitude $R=d$, then for all ideals $I$ in $R$, every minimal prime divisor of $u \mathscr{R}(R, I)$, of $(u \mathscr{R}(R, I))_{a}$, and of $u \mathscr{R}(R, I)^{\prime}$ has depth $=d$.

Proof. This holds for $u \mathscr{R}(R, I)^{\prime}$, by (4.6), so it holds for $u \mathscr{R}(R, I)$ and $(u \mathscr{R}(R, I))_{a}$, by integral dependence.

The last application that will be given is related to the (catenary) chain conjectures.

(8.6) Proposition. Let $Q$ be an open ideal in a semi-local domain $R$, let $\mathscr{R}=\mathscr{R}(R, Q)$, and let $\mathscr{R}^{0}=\mathscr{R}\left(R^{*}, Q R^{*}\right)$. Then $\{P \in \operatorname{Spec} \mathscr{R}$; $u \in P$, height $P>1$, and there exists a height one maximal ideal in $\left.\left(\mathscr{R}_{P}\right)^{\prime}\right\}=\left\{P^{0} \cap \mathscr{R} ; P^{0} \in \mathrm{Spec} \mathscr{R}^{0}\right.$, height $P^{0}>1$, and $P^{0}$ is a minimal prime divisor of $\left(u, z^{*}\right) \mathscr{R}^{0}$, for some minimal prime ideal $z^{*}$ in $\left.\mathscr{R}^{0}\right\}$.

Proof. Let the first set be $E_{1}$ and the second set $E_{2}$. Let $P \in E_{1}$, so $u \in P$, and so $P^{0}=P \mathscr{R}^{0}$ is prime and $\mathscr{R}_{P}$ is a dense subspace of $\mathscr{R}_{P^{0}}^{0}$ (4.1). Also, there exists a depth one minimal prime ideal in the completion of $\mathscr{R}_{P}$ (by [9, Proposition 3.5], since $P \in E_{1}$ ), so there exists a depth one minimal prime ideal in the completion of $\mathscr{R}_{P^{0}}^{0}$. Therefore there exists a depth one minimal prime ideal in $\mathscr{R}_{P^{0}}^{0}$, by (6.5.2). Thus $P^{0}$ is a minimal prime divisor of $\left(u, z^{*}\right) \mathscr{R}^{0}$, for some minimal prime ideal $z^{*}$ in $\mathscr{R}^{0}$, so $P \in E_{2}$, since $P=P^{0} \cap \mathscr{R}$.

On the other hand, if $P \in E_{2}$ and $P^{0}$ is the prime ideal in $\mathscr{R}^{0}$ such that $P^{0} \cap \mathscr{R}=P$, then $\mathscr{R}_{P}$ is a dense subspace of $\mathscr{R}_{P^{0}}^{0}(4.1)$. Also, there exists a depth one minimal prime ideal in $\mathscr{R}_{P^{0}}^{0}$, so there exists a depth one minimal prime ideal in the completion of $\mathscr{R}_{P}$, hence there exists a height one maximal ideal in the integral closure of $\mathscr{R}_{P}[\mathbf{9}$, Proposition 3.5], and so it follows that $P \in E_{1}$.

The equal sets in (8.6) are finite, since each $\left(u, z^{*}\right) \mathscr{R}^{0}$ has only finitely many minimal prime divisors and there are only finitely many $z$ *.

9. Three questions. There are many questions concerning prime divisors of zero in form rings which haven't been answered in this 
paper. The following three are certainly not the most important, but answers to them would be of some interest.

(9.1) Question. ${ }^{1} \quad$ Let $P$ a prime ideal in a Noetherian ring $A$ and let $\mathscr{R}=\mathscr{R}(A, P)$. If $u \mathscr{R}$ is primary and integrally closed, is $u \mathscr{R}$ prime?

Concerning (9.1), the following result has appeared in two recent papers: $u \mathscr{R}$ is prime if and only if $\mathscr{F}\left(A_{P}, P A_{P}\right)$ is a domain and $P^{n}$ is $P$-primary, for all $n \geqq 1$ [2, Theorem 1] and [17, Corollary 1.2]. Now, if $u \mathscr{R}$ is prime, then $u \mathscr{R}$ is integrally closed (6.4.2) and primary. Moreover, it follows from any one of $[\mathbf{5},(25.15)],[\mathbf{7}$, Theorem 5], or [21, Theorem 2, p. 250] that $A_{P}$ is analytically irreducible. This last also holds if $u \mathscr{R}$ is primary and integrally closed (by using (5.1.1), (6.2.5), (6.2.7), (6.4.2), and (5.2.1) applied to $\mathscr{R}\left(A_{P}, P A_{P}\right)$ and $\left.\mathscr{R}\left(A_{P}^{*}, P A_{P}^{*}\right)\right)$. But I don't know if it implies that $u \mathscr{R}$ is prime.

(If $P$ isn't required to be prime in (9.1), then it is easy to see that the answer is no: let $(R, M)$ be a regular local ring and let $P=M^{k}$ (for a fixed $k>1$ ), so $u \mathscr{R}$ is integrally closed (by (6.2.5), since $P$ is normal (since $M$ is)) and is primary (by (6.4.2), since it has only one minimal prime divisor (5.3)), but $u \mathscr{R}$ isn't prime, since $u \mathscr{R} \cap R$ isn't prime. Also, see (8.3).)

(9.2) Question. For what local rings $(R, M)$ does there exist an $M$-primary ideal $Q$ such that the number of prime divisors of $u \mathscr{R}(R, Q)^{\prime}$ is equal to the number of minimal prime ideals in $R^{*}$, and what properties hold for the rings in this class of rings?

Note that every regular local ring has this property. (Also, see (8.3).)

In [5, (25.1)], M. Nagata shows that if all prime divisors of zero in $\mathscr{R}(R, Q)$ have the same depth, where $Q$ is an open ideal in a local ring $R$, then $R$ is unmixed. The last question concerns the converse of this.

(9.3) Question. If $R$ is an unmixed local ring, does there exist an open ideal $Q$ in $R$ such that every prime divisor of $u \mathscr{R}(R, Q)$ has depth = altitude $R$ ?

If $R$ is also analytically unramified in (9.3), then the answer is seen to

\footnotetext{
1 The following example, due to the referee, shows that the answer is no. Let $C$ be the field of complex numbers, and let $A=C[[X, Y, Z]] / K$, where $K=\left(X^{2}+Y^{3}+Z^{5}\right)$. Then $A$ is a $U F D$, by Theorem 25.1 in [J. Lipman, Rational Singularities with Applications to Algebraic Surfaces and Unique Factorization, Inst. Hautes Etudes Sci. Publ. Math., No. 36, Presses Universitaires de France, Paris, France, 1969, pp. 195-279], so $A=A^{\prime}$. Also, the maximal ideal $M$ in $A$ is a normal ideal, by Theorem 7.1 [Ibid.], so $\mathscr{R}=\mathscr{R}(A, M)=\mathscr{R}^{\prime}$, by $(2.6 .2)$, hence $u \mathscr{R}=(u \mathscr{R})_{\alpha} . \quad$ Finally, $\mathscr{R} / u \mathscr{R} \cong(2.2 .5)$ $\mathscr{F}(A, M) \cong C[X, Y, Z] /\left(X^{2}\right) \quad$ (since $(X+K)^{2} \in M^{3}$ implies $(t(X+K))^{2} \in u \mathscr{R}$, so $\operatorname{Rad} u \mathscr{R}=$ $(u, t(X+K)) \mathscr{R}$ is prime (by the structure of $\mathscr{R} /(u, t(X+K)) \mathscr{R}))$, so $u \mathscr{R}$ is primary and integrally closed, but not prime.
} 
be yes, by letting $Q$ be the ideal $B$ of (7.1). (For then, $u \mathscr{R}=(u \mathscr{R})_{a}$ (6.2.6), so each prime divisor of $u \mathscr{R}$ has height one (6.4.2), so the conclusion follows from (7.5).)

\section{REFERENCES}

1. M. F. Atiyah and I. G. Macdonald, Introduction to Commutative Algebra, Addison-Wesley, Reading, Mass., 1969.

2. M. Hochster, Criteria for equality of ordinary and symbolic powers of primes, Math. Z., 133 (1973), 53-65.

3. W. Krull, Dimensionstheorie in Stellenringen, J. Reine Angew. Math., 179 (1938), 204-226.

4. M. Nagata, On the chain problem of prime ideals, Nagoya Math. J., 10 (1965), 51-64.

5. Local Rings, Interscience Tracts 13, Interscience, New York, 1962.

6. D. G. Northcott, Ideal Theory, Cambridge Tracts 42, Cambridge University Press, Cambridge, 1953.

7. - On the notion of a form ideal, Quart. J. Math., 4 (1953), 221-229.

8. J. W. Petro, Some results on the asymptotic completion of an ideal, Proc. Amer. Math. Soc., 15 (1964), 519-524.

9. L. J. Ratliff, Jr., On quasi-unmixed local domains, the altitude formula, and the chain condition for prime ideals (I), Amer. J. Math., 91 (1969), 508-528.

10. - On quasi-unmixed local domains, the altitude formula, and the chain condition for prime ideals (II), Amer. J. Math., 92 (1970), 99-144.

11. On prime divisors of the integral closure of a principal ideal, J. Reine Angew. Math., 255 (1972), 210-220.

12. Locally quasi-unmixed Noetherian rings and ideals of the principal class, Pacific J. Math., 52 (1974), 185-205.

13. D. Rees, Valuations associated with a local ring (I), Proc. London Math. Soc., 5 (1955), $107-128$.

14. - Valuations associated with a local ring (II), J. London Math. Soc., 31 (1956), 228-235.

15. - A note on form rings and ideals, Mathematika, 4 (1957), 51-60.

16. - The grade of an ideal or module, Proc. Cambridge Philos. Soc., 53 (1957), 28-42.

17. L. Robbiano and G. Valla, Primary powers of a prime ideal, Pacific J. Math., 63 (1976), 491-498.

18. M. Sakuma and H. Okuyama, On a criterion for analytically unramification of a local ring, J. Gakugei Tokushima Univ., 15 (1966), 36-38.

19. P. G. Sawtelle, Characterizations of unmixed and quasi-unmixed local domains, Ph.D. Thesis, University of California, Riverside, 1971.

20. M. Smith, Strongly superficial elements, Pacific J. Math., 58 (1975), 643-650.

21. O. Zariski and P. Samuel, Commutative Algebra, Vol. II, Van Nostrand, New York, 1960.

Received January 12, 1977. Research on this paper was supported in part by the National Science Foundation Grant MCS 76-06009.

UNIVERSITY OF CALIFORNIA

RIVERSIDE, CA 92502 



\section{PACIFIC JOURNAL OF MATHEMATICS \\ EDITORS}

RICHARD ARENS (Managing Editor)

University of California

Los Angeles, CA 90024

\section{R. A. Beaumont \\ University of Washington \\ Seattle, WA 98105}

C. C. MOORE

University of California

Berkeley, CA 94720

\section{J. DUGUNDJI}

Department of Mathematics

University of Southern California

Los Angeles, CA 90007

R. FINN AND J. MILGRAM

Stanford University

Stanford, CA 94305

\section{ASSOCIATE EDITORS}

\section{E. F. BECKENBACH}

B. H. NeUmanN

F. WOLF

K. YoSHIDA

\section{SUPPORTING INSTITUTIONS}

\author{
UNIVERSITY OF BRITISH COLUMBIA \\ CALIFORNIA INSTITUTE OF TECHNOLOGY \\ UNIVERSITY OF CALIFORNIA \\ MONTANA STATE UNIVERSITY \\ UNIVERSITY OF NEVADA \\ NEW MEXICO STATE UNIVERSITY \\ OREGON STATE UNIVERSITY \\ UNIVERSITY OF OREGON \\ OSAKA UNIVERSITY
}

\author{
UNIVERSITY OF SOUTHERN CALIFORNIA \\ STANFORD UNIVERSITY \\ UNIVERSITY OF HAWAII \\ UNIVERSITY OF TOKYO \\ UNIVERSITY OF UTAH \\ WASHINGTON STATE UNIVERSITY \\ UNIVERSITY OF WASHINGTON \\ AMERICAN MATHEMATICAL SOCIETY
}

The Supporting Institutions listed above contribute to the cost of publication of this Journal, but they are not owners or publishers and have no responsibility for its contents or policies.

Mathematical papers intended for publication in the Pacific Journal of Mathematics should be in typed form or offset-reproduced (not dittoed), double spaced with large margins. Underline Greek letters in red, German in green, and script in blue. The first paragraph or two must be capable of being used separately as a synopsis of the entire paper. Items of the bibliography should not be cited there unless absolutely necessary, in which case they must be identified by author and Journal, rather than by item number. Manuscripts, in duplicate, may be sent to any one of the four editors. Please classify according to the scheme of Math. Reviews, Index to Vol. 39. All other communications should be addressed to the managing editor, or Elaine Barth, University of California, Los Angeles, California, 90024.

100 reprints are provided free for each article, only if page charges have been substantially paid. Additional copies may be obtained at cost in multiples of 50 .

The Pacific Journal of Mathematics is issued monthly as of January 1966. Regular subscription rate: $\$ 72.00$ a year (6 Vols., 12 issues). Special rate: $\$ 36.00$ a year to individual members of supporting institutions.

Subscriptions, orders for back numbers, and changes of address should be sent to Pacific Journal of Mathematics, 103 Highland Boulevard, Berkeley, California, 94708.

PUBLISHED BY PACIFIC JOURNAL OF MATHEMATICS, A NON-PROFIT CORPORATION

Printed at Jerusalem Academic Press, POB 2390, Jerusalem, Israel.

Copyright (C) 1977 Pacific Journal of Mathematics All Rights Reserved 


\section{Pacific Journal of Mathematics \\ Vol. 70, No. $2 \quad$ October, 1977}

B. Arazi, A generalization of the Chinese remainder theorem ........... 289

Thomas E. Armstrong, Polyhedrality of infinite dimensional cubes .... . . . 297

Yoav Benyamini, Mary Ellen Rudin and Michael L. Wage, Continuous

images of weakly compact subsets of Banach spaces ............ 309

John Thomas Burns, Curvature functions on Lorentz 2-manifolds ......... 325

Dennis F. De Riggi and Nelson Groh Markley, Shear distality and equicontinuity .................................. 337

Claes Fernström, Rational approximation and the growth of analytic

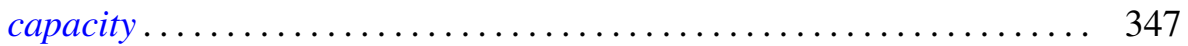

Pál Fischer, On some new generalizations of Shannon's inequality....... 351

Che-Kao Fong, Quasi-affine transforms of subnormal operators ......... 361

Stanley P. Gudder and W. Scruggs, Unbounded representations of

*-algebras........................................ 369

Chen F. King, A note on Drazin inverses .................... 383

Ronald Fred Levy, Countable spaces without points of first countability . . . 391

Eva Lowen-Colebunders, Completeness properties for convergence

spaces ......................................... 401

Calvin Cooper Moore, Square integrable primary representations ....... 413

Stanisław G. Mrówka and Jung-Hsien Tsai, On preservation of

E-compactness ................................ 429

Yoshiomi Nakagami, Essential spectrum $\Gamma(\beta)$ of a dual action on a von

Neumann algebra ................................ 437

L. Alayne Parson, Normal congruence subgroups of the Hecke groups

$G\left(2^{(1 / 2)}\right)$ and $G\left(3^{(1 / 2)}\right)$...

Louis Jackson Ratliff, Jr., On the prime divisors of zero in form rings . . . . 489

Caroline Series, Ergodic actions of product groups .................. 519

Robert O. Stanton, Infinite decomposition bases..................... 549

David A. Stegenga, Sums of invariant subspaces .................. 567 